\title{
BORDISMO DE AÇÕES NÃO-SINGULARES
}

\author{
Valter Locci
}

Orientador: Prof. Dr. Janey Antonio Daccach

Tese de Doutorado apresentada ao Instituto de Ciências Matemáticas e de Computação, da Universidade de São Paulo, como parte dos requisitos para obtenção do título de Doutor em Ciências - Área: Matemática - Geometria e Topologia.

USP - São Carlos

1998 
À minha esposa Marise e aos meus filhos Bruna e Rafael. 


\section{AGRADECIMENTOS}

Primeiramente. a Deus por tudo. E, em especial, por ter proporcionado-me mais um período de desenvolvimento intelectual.

Ao meu orientador pela orientação.

À minha esposa pela compreensão e dedicação.

À CAPES-PICD pelo auxílio financeiro.

Agradeço, enfim, a todos que contribuíram direta ou indiretamente com este trabalho. 


\section{RESUMO}

Dada uma ação de um grupo de Lie $G$ numa variedade $M$, uma construção geométrica, chamada blow-up, é utilizada para obter uma nova variedade denotada por $B(A, M)$, onde $A$ é um certo subconjunto invariante de $M$.

Quando $G$ é abeliano, através de uma sequiência finita de tais blow-ups equivariantes, uma nova variedade $M^{\prime}$ é obtida, dotada de uma ação não-singular de $G$.

Neste trabalho estudamos em que condições a variedade $M^{\prime}$ pertence à mesma classe de bordismo de $M$, e também alguns resultados sobre bordismo de açōes não-singulares são obtidos. 


\begin{abstract}
Given an action of a Lie Group $G$ on a manifold $M$, a geometric construction, called blow-up, is performed to yield a new manifold denoted by $B(A, M)$, where $A$ is a certain invariant subset of $M$.

When $G$ is abelian, by a finite sequence of such equivariant blow-ups, a new manifold $M^{\prime}$ is obtained, carriyng a nonsingular action of $G$.

In this work we study under which condictions the manifold $M^{\prime}$ belongs to the same cobordism class of $M$, and also some results on cobordism of nonsingular actions are obtained.
\end{abstract}




\section{Índice}

Introdução

1 Grupos de Transformação e Fibrados 1

1.1 Grupos de Transformação . . . . . . . . . . . . . . . 1

1.2 Tipos de Órbita e Tipos de Isotropia $\ldots \ldots \ldots \ldots \ldots$

1.3 Órbita Principal e Tipo de Isotropia Principal . . . . . . . . 8

1.4 Fibrados . . . . . . . . . . . . . . . . . 9

1.5 Fibrado Universal (Espaços Classificantes) $\ldots \ldots \ldots \ldots \ldots$

2 Bordismo e Números de Stiefel-Whitney 18

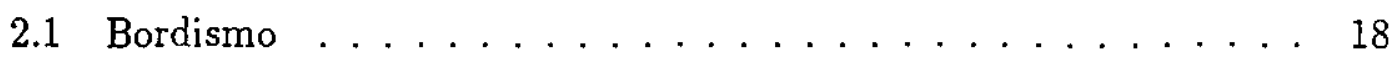

2.2 Classes de Stiefel-Whitney . . . . . . . . . . . . . . . 22

2.3 Números de Stiefel-Whitney . . . . . . . . . . . . . . 27

2.4 Fibrado Involução . . . . . . . . . . . . . . . . . . . . . 29

2.5 Classe de Bordismo de Variedade com Involução . . . . . . . . 34

2.6 Fibrado Linha Gerado por uma Subvariedade 1-Codimensional . . 36

3 Blow-up e Ações Não-Singulares 42

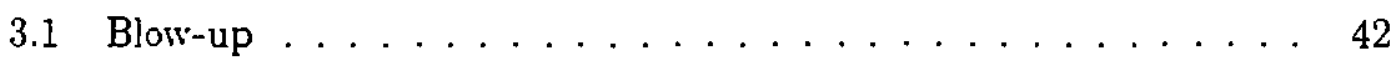

3.2 Ações Não-Singulares . . . . . . . . . . . . . . . . 52

3.3 Classes de Bordismo de $\mathrm{M}$ e $\mathrm{B}(\mathrm{A}, \mathrm{M}) \ldots \ldots \ldots \ldots$

3.4 Bc:dismo de Variedades Não-Singulares . . . . . . . . . . . 62

$\begin{array}{ll}\text { Bibliografia } & 66\end{array}$ 


\section{Introdução}

Conner e Floyd em [4] mostram que se $M$ é uma $\mathbb{Z}_{2}$-variedade diferenciável fechada e $\nu=\nu(F, M)$ é o fibrado normal sobre o conjunto de pontos fixos $F$, então $M$ e $\mathbb{R} P(\nu \oplus 1)$ bordam, isto é, $[M]_{2}=[\mathbb{R P}(\nu \oplus 1)]_{2}$.

Mais geralmente, considerando $G$ um grupo de Lie compacto e uma $G$-variedade $M$, podemos perguntar em que casos tal relação se generaliza. Mais ainda, em caso negativo, quais outros relacionamentos possíveis entre a classe de bordismo de $M$ e outras variedades relacionadas com os pontos fixos $F$.

Wasserman em [14], considerando uma adequada subvariedade invariante $A$ de uma $G$-variedade $M$, define o blow-up de $A$ em $M$ como sendo

$$
B(A, M)=\underset{A}{\#} \mathbb{R} P(\nu \oplus 1),
$$

onde $\#_{A}$ é a soma conexa ao longo de $A$, e o utiliza simplificar a estrutura de órbitas de $M$. No caso de $G$ ser abeliano ele obtém certas variedades chamadas de não-singulares.

Posteriormente, ao analisar o relacionamento entre certas classes caracteristicas e o conjunto de pontos fixos, Wasserman, em [15], constrói uma variedade $W$ cujo bordo é a união disjunta de três das variedades da definição do blow-up, isto é,

$$
\partial W=M \dot{U} B(A, M) \dot{U} \mathbb{R} P(\nu \oplus 1)
$$


Segue imediatamente desta construção de Wasserman o seguinte relacionamento entre as classes de bordismo das variedades envolvidas:

$$
[B(A, M)]_{2}=[M]_{2}+[\operatorname{RPP}(\nu \oplus 1)]_{2} .
$$

Neste trabalho, considerando o blow-up de todos os pontos fixos $F$ de uma $G$-variedade $M$ obtemos um relacionamento geral envolvendo as classes de bordismo de $M$ e de $\mathbb{R} P(\nu \oplus 1)$ que depende da classe do blow-up, isto é,

$$
[B(F, M)]_{2}=[M]_{2}+[\operatorname{RRP}(\nu \oplus 1)]_{2} .
$$

Impondo restrições sobre $G$ e sobre o conjunto de pontos fixos $F$ obtemos relacionamentos mais específicos entre as classes de bordismo de $M$ e de $B(F, M)$. Em especial, quando $M$ é uma $\mathbb{Z}_{2}$-variedade não-singular, obtemos pelo Teorema de Conner-Floyd que $[B(F, M)]_{2}=[M]_{2}=0$. Destes resultados obtemos algumas informações já conhecidas sobre a não existência de involuções com certos tipos de pontos fixos pré-determinados.

Nos Capítulos I e II, após introduzir a notação e enunciar os resultados básicos sobre Grupos de Transformação, Fibrados, Bordismo e Números de Stiefel-Whitney, chegando ao Teorema de Conner Floyd, obtemos uma condição para que uma $\mathbb{Z}_{2}$-variedade $M$ com conjunto de pontos fixos 1-codimensional tenha classse de bordismo nula.

No Capítulo III, após definir e exemplificar as noções de blow-up e de variedades não-singulares, estabelecemos o exposto acima. 


\section{Capítulo 1}

\section{Grupos de Transformação e Fibrados}

Neste capítulo introduzimos a notação e alguns fatos básicos sobre grupos de transformação e fibrados. As provas destes resultados podem ser encontradas em [6] e [11].

\subsection{Grupos de Transformação}

Definição 1.1.1. Seja G um grupo topológico e $X$ um espaço topológico de Hausdorff. Uma G-ação é uma aplicação contínua $\varphi: G \times X \rightarrow X$ tal que:

1) $\varphi(e, x)=x$ para todo $x \in X$, onde $e$ é a identidade de $G$;

2) $\varphi\left(g_{2}, \varphi\left(g_{1}, x\right)\right)=\varphi\left(g_{2} g_{1}, x\right)$ para todo $g_{1}, g_{2} \in G$ e $x \in X$.

A tripla $(X, G, \varphi)$ é chamada de grupo de transformação topológico ou $G$ ação sobre $X$, e $X$ é chamado de G-espaço.

Quando $\varphi$ é compreendida do contexto, usamos a notação

$$
\varphi(g, x)=g \cdot x=g x .
$$

Daí, as condições acima são descritas como:

$$
\begin{aligned}
& \text { 1) } e x=x \\
& \text { 2) } g_{2}\left(g_{1} x\right)=\left(g_{2} g_{1}\right) x
\end{aligned}
$$


Quando $G$ é fixado, denotamos $(X, G, \varphi)$ simplesmente por $X$.

Para cada $g \in G$ temos uma aplicação bijetora

$$
\varphi_{g}: X \rightarrow X
$$

definida por $x \rightarrow \varphi_{g}(x)=\varphi(g, x)$, que é um homeomorfismo de $X$.

Se $G$ é um grupo de Lie, $X$ é uma variedade diferenciável e $\varphi$ é aplicação diferenciável, então $\varphi_{g}$ é um difeomorfismo.

Dado um $G$-espaço $X$ denotamos:

$$
\begin{aligned}
G(x) & =\{g x \in X \mid g \in G\} \quad \text { órbita de } x \in X \\
G_{x} & =\{g \in G \mid g x=x\} \quad \text { grupo de isotropia de } x \\
X^{G} & =\operatorname{Fix}(G, X)=\left\{x \in X \mid G_{x}=G\right\} \quad \text { conjunto dos pontos fixos. }
\end{aligned}
$$

Note que se $g \in G$ e $x \in X$, então $G_{g x}$ e $G_{x}$ são conjugados, isto é,

$$
G_{g x}=g G_{x} g^{-1}
$$

Definição 1.1.2. Dados dois G-espaços $X$ e $Y$, uma aplicação $f: X \rightarrow Y$ é chamada $G$-aplicação ou aplicação equivariante se para cada $g \in G$ e $x \in X$ temos

$$
f(g x)=g f(x)
$$

Uma $G$-aplicação que é bijetora é chamada $G$-equivalên cia. Quando existe uma $G$-equivalência $\quad f: X \rightarrow Y$ dizemos que $X$ e $Y$ são $G$-equivalentes e denotamos $X \cong Y$.

Considerando $Y$ um subconjunto de um $G$-conjunto $X$ denotamos

$$
g Y=\{g y \mid y \in Y\}
$$

Dizemos que o subconjunto $Y$ é $G$-invariante se $g Y \subset Y, \forall g \in G$. Claramente um subconjunto invariante $Y$ de um $G$-espaço $X$ satisfaz, $\forall g \in G, g Y=Y$. Naturalmerte $G(x)$ é um subconjunto $G$-invariante de $X$. 


\section{Denotamos}

$$
\begin{aligned}
& \frac{X}{G}=\{G(x) \mid x \in G\} \quad \text { conjunto das órbitas } \operatorname{de} X . \\
& \pi: X \rightarrow \frac{X}{G} \text { aplicação natural levando } x \operatorname{em} G(x) .
\end{aligned}
$$

O conjunto $\frac{X}{G}$ dotado da topologia quociente é chamado de espaço das órbitas de X.

Se $X$ é um $G$-espaço e $x \in X$ existe uma aplicação natural

$$
\alpha_{x}: \frac{G}{G_{x}} \rightarrow G(x)
$$

definida por $\alpha_{x}\left(g G_{x}\right)=g x$.

Proposição 1.1.3. Se Gé compacto então $\alpha_{x}: \frac{G}{G_{x}} \rightarrow G(x)$ é um homeomorfismo.

Definição 1.1.4. Um G-espaço X é chamado:

1. trivial se $G_{x}=G, \forall x \in X$;

2. livre se $G_{x}=\{e\}, \forall x \in X$;

3. semi-livre se $G_{x}=\{e\}$ ou $G_{x}=G, \forall x \in X$;

4. transitivo se existe apenas uma órbita, isto é $G(x)=X$;

5. efetivo se $\bigcap_{x \in X} G_{x}=\{e\}$.

A proposição abaixo caracteriza $G$-aplicações.

Proposição 1.1.5. Sejam $G$ um grupo compacto e $H, K$ dois subgrupos fechados de G. Então

1. existe uma $G$-aplicação $f: \frac{G}{H} \rightarrow \frac{G}{K} \quad \Leftrightarrow \quad H$ é conjugado a um subgrupo de $K$;

2. se existe $a \in G$ com $a H a^{-1} \subset K$ então a aplicação $f_{a}: \frac{G}{H} \rightarrow \frac{G}{K}$ definida por $g H \rightarrow f_{a}(g H)=g a^{-1} K$ é G-aplicação e toda G-aplicação tem esta forma. 


\subsection{Tipos de Órbita e Tipos de Isotropia}

Assumimos aqui que $G$ é um grupo topológico compacto e que todos os espaços são de Hausdorff.

Denotemos por $\mathcal{F}$ a família de todos os $G$-espaços homogêneos (ação transitiva) de Hausdorff. Considere em $\mathcal{F}$ a relação de equivalência $\sim$ dada por:

$$
X \sim Y \Leftrightarrow \text { existe um G-homeomorfismo } f: X \rightarrow Y .
$$

Denote por $(X)$ a classe de equivalência contendo $X$.

Definição 1.2.1. A classe $(X)$ é chamada tipo de órbita e o conjunto de tais classes $\frac{\mathcal{F}}{\sim}$ é chamado conjunto dos tipos de órbitas.

Segue da Proposição 1.1.3 que para cada $G$-espaço homogêneo de Hausdorff $X$, existe um subgrupo fechado $H$ de $G$ tal que são $G$-homeomorfos

$$
X \cong \frac{G}{H}
$$

isto é, cada $(X)$ é representado por um espaço de classes laterais $\frac{G}{H}$.

Um ordenamento parcial do conjunto $\frac{\mathcal{F}}{\sim}$ é dado por

$$
(X) \geq(Y) \Leftrightarrow \text { existe uma G-aplicação } f: X \rightarrow Y \text {. }
$$

Neste conjunto ordenado parcialmente temos:

$$
\begin{aligned}
& \left(\frac{G}{e}\right) \text { é um máximo } \\
& \left(\frac{G}{G}\right) \text { é um mínimo. }
\end{aligned}
$$

Definição 1.2.2. Quando um $G$-espaço homogêneo $X$ é equivalente a $\frac{G}{H}$, denotamos a classe de conjugação de $H$ em $G$ por $(H)$, isto é,

$$
H_{\mathrm{I}}=\{K \subset G ; K \sim H\}=\left\{K \subset G ; K=g H g^{-1}, g \in G\right\}
$$

e chamamos $(H)$ de o tipo de isotropia de $X$. 
Em particular, se $G$ é abeliano temos $(H)=H$.

Genericamente, dado um $G$-espaço $X$, uma órbita que é equivalente a $\frac{G}{H}$ é chamada de:

$$
\begin{gathered}
\text { órbita com tipo de isotropia }(H) \\
\text { órbita com tipo de órbita }\left(\frac{G}{H}\right) \text {. }
\end{gathered}
$$

Um ordenamento parcial das classes de conjugação de subgrupos fechados de $G$ é dado por

$$
(H) \leq(K) \Leftrightarrow H \text { é conjugado a um subgrupo de } K \text {. }
$$

Resumidamente temos então os dois ordenamentos parciais:

$$
\begin{gathered}
\left(\frac{G}{H}\right) \geq\left(\frac{G}{K}\right) \Leftrightarrow \exists f: \frac{G}{H} \rightarrow \frac{G}{K}, G-\text { aplicação } \stackrel{1.1 .5}{\Longrightarrow} \exists a \in G \mid a H a^{-1} \subset K \\
(H) \leq(K) \Leftrightarrow H \sim K^{\prime}, K^{\prime} \subset K \Leftrightarrow \exists a \in G \mid a H a^{-1} \subset K .
\end{gathered}
$$

Assim

$$
\left(\frac{G}{H}\right) \geq\left(\frac{G}{K}\right) \Leftrightarrow(H) \leq(H)
$$

isto é,

Proposição 1.2.3. A correspondência $\left(\frac{G}{H}\right) \rightarrow H$ é um anti-isomorfismo do conjunto parcialmente ordenado $\underset{\sim}{\sim}$ no conjunto parcialmente ordenado das classes de conjugação dos subgrupos fechados de $G$.

Quando $(e)$ e $(G)$ são tipos de isotropia do $G$-espaço $X$, esta correspondência poder ser representada por

Tipo de G-órbitas: $\left(\frac{G}{e}\right) \geq \ldots \geq\left(\frac{G}{H}\right) \geq\left(\frac{G}{K}\right) \geq \ldots \geq\left(\frac{G}{G}\right)$

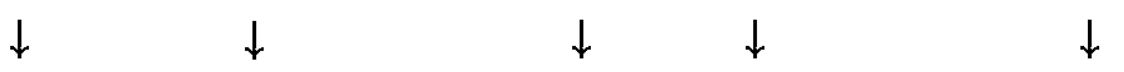

Tipo de isotropia: $(e) \leq \ldots \leq(H) \leq(K) \leq \ldots \leq(G)$. 
Dado $X$ um $G$-espaço e $H$ um subgrupo de $G$ denotamos por $X(H)$ a união das órbitas de $X$ com tipo de isotropia $(H)$, e por $X(\geq H)$ a união das órbitas de $X$ com tipo de isotropia maior ou igual a $(H)$, isto é,

$$
\begin{aligned}
X(H) & =\left\{x \in X ;\left(G_{x}\right)=(H)\right\}=\left\{x \in X ; G_{x} \sim H\right\} \\
& =\left\{x \in X ; \exists a \in G \mid G_{x}=a H a^{-1}\right\} \\
X(\geq H) & =\left\{x \in X ; G_{x} \supset H^{\prime} \sim H\right\}=\left\{x \in X ; \exists a \in G \mid G_{x} \supset a H a^{-1}\right\} .
\end{aligned}
$$

Em particular temos:

$$
\begin{aligned}
X(G) & =X^{G} & & \text { pontos fixos } \\
X(e) & =\left\{x \in X ; G_{x}=e\right\} & & \text { pontos "livres". }
\end{aligned}
$$

Proposição 1.2.4. $G X^{H}=X(\geq H)$.

Corolário 1.2.5. Se $G$ é compacto e $X$ é um G-espaço de Hausdorff então $X(\geq$ H) é fechado em $X$.

Corolário 1.2.6. Sejam $G$ compacto e $X$ um G-espaço de Hausdorff. Se (H) é maximal entre os tipos de isotropia que aparecem em $X$, então $X(H)$ é fechado em $X$.

Dado um $G$-espaço $X$ temos uma decomposição de $X$ como união disjunta dos subconjuntos $X(H)$, isto é,

$$
X=\bigcup_{(H)} X(H),
$$

onde $(H)$ :aria sobre todas as classes de conjugação de subgrupos de $G$.

Em geral $\mathrm{X}(H)$ não é fechado em $X$. Mas em casos especiais podemos ordenar e agrupar is $X(H)$ em subconjuntos fechados. 
Proposição 1.2.7. Dadas finitas classes de conjugação $\left(H_{1}\right), \ldots,\left(H_{n}\right)$ de subgrupos fechados de $G$, podemos arranjá-las tal que

$$
\left(H_{i}\right) \geq\left(H_{j}\right) \Rightarrow i \leq j
$$

Em particular, quando $G$ é abeliano temos:

$$
H_{i} \supset H_{j} \Leftrightarrow\left(H_{i}\right) \geq\left(H_{j}\right) \Rightarrow i \leq j .
$$

Proposição 1.2.8. Seja $G$ um grupo compacto e $X$ um G-espaço de Hausdorff. Suponha que $\left\{\left(H_{i}\right)\right\}$, conjunto dos tipos de isotropia aparecendo em $X$, é finito. Ordene-o como na Proposição 1.2.7. Se denotarmos

$$
X_{i}=X\left(H_{1}\right) \cup \cdots \cup X\left(H_{i}\right)
$$

então $X_{i}$ é fechado em $X$.

Dado um $G$-espaço $X$, dizemos que $X$ tem $\mathbf{n}$ tipos de órbita quando existem exatamente $\mathrm{n}$ tipos de órbitas diferentes em $X$.

Teorema 1.2.9. Se $G$ é um grupo de Lie compacto e $M$ é uma G-variedade compacta, então existem apenas finitos tipos de órbita em $M$. 


\section{3 Órbita Principal e Tipo de Isotropia Prin- cipal}

Como já vimos anteriormente, entre os tipos de órbita a menor é $\frac{G}{G}$ e a maior é $\frac{G}{\{e\}}$. Mas estes tipos de órbitas nem sempre ocorrem em um $G$-espaço $X$.

O teorema abaixo afirma a existência de um tipo de órbita máximo quando $M / G$ é conexo. Relembramos que $M(H)$ denota a união das órbitas de tipo $G / H$.

Teorema 1.3.1. Seja $G$ um grupo de Lie compacto e $M$ uma G-variedade. Se o espaço das órbitas $\frac{M}{G}$ é conexo, então existe um tipo de órbita máximo $\frac{G}{H}$ em $M$. Mais ainda,

$$
\begin{aligned}
& M(H) \text { é aberto e denso em } M ; \\
& \frac{M(H)}{G} \text { é conexo. }
\end{aligned}
$$

Definição 1.3.2. O tipo de órbita máximo garantido pelo teorema acima é chamado de tipo de órbita principal e as órbitas deste tipo são chamadas de órbitas principais. Os grupos de isotropias correspondentes säo chamados de grupos de isotropia principais. 


\subsection{Fibrados}

Aqui vamos definir, construir e enunciar alguns resultados básicos envolvendo fibrados que serão utilizados no que segue. As provas podem ser encontradas em Milnor [11] e em Kawakubo [6].

Dado uma $G$-ação $\varphi: G \times X \rightarrow X$ associamos a cada $g \in G$ a aplicação $\varphi_{g}: X \rightarrow X$ dada por $\varphi_{g}(x)=\varphi(g, x)$, que é um homeomorfismo de $X$ em $X, \mathrm{e}$ definimos um homomorfismo $\Phi: G \rightarrow \operatorname{Homeo}(X)$ por $g \rightarrow \Phi_{g}=\varphi_{g}$.

Quando a ação é efetiva temos que $\Phi$ é um monomorfismo. Assim identificaremos $G \cong \Phi(G) \subset$ Homeo $(X)$, e denotaremos simplesmente $G \subset$ Homeo $(X)$.

Definição 1.4.1 (Fibrado Coordenadas). Seja $K$ um grupo topológico atuando efetivamente em um espaço topológico F. Sejam $E$ e $X$ espaços de Hausdorff e $\pi: E \rightarrow X$ uma aplicação contínua. Suponha que existam uma cobertura aberta $\left\{U_{\alpha}\right\}_{\alpha \in \Lambda}$ de $X$ e um homeomorfismo $\varphi_{\alpha}: \pi^{-1}\left(U_{\alpha}\right) \rightarrow U_{\alpha} \times F$ para cada $\alpha \in \Lambda$ tendo as três propriedades seguintes:

1. $p_{\alpha} \cdot \varphi_{\alpha}=\pi$, onde $p_{\alpha}: U_{\alpha} \times F \rightarrow U_{\alpha}$ é a projeção;

2. Defina, para cada $x \in U_{\alpha}, \quad \varphi_{\alpha, x}: \pi^{-1}(x) \rightarrow F$ por $z \rightarrow \varphi_{\alpha, x}(z)=p_{\alpha}^{\prime} \cdot \varphi_{\alpha}(z)$, onde $p_{\alpha}^{\prime}: U_{\alpha} \times F \rightarrow F$ é a outra projeção. Denote $\Theta_{\beta \alpha}(x)=\varphi_{\beta, x} \cdot \varphi_{\alpha, x}^{-1}$ para $x \in U_{\alpha} \cap U_{\beta}$. Entäo $\Theta_{\beta \alpha}(x) \in K \subset \operatorname{Homeo}(F)$.

3. A aplicação $\Theta_{\beta \alpha}: U_{\alpha} \cap U_{\beta} \rightarrow K$ assim obtida é contínua.

Então, juntando as informações acima, o sistema $\left(E, \pi, X, F, K, U_{\alpha}, \varphi_{\alpha}\right)$ é chamado de um fibrado coordenadas.

Definição 1.4.2 (Fibrado). Dois fibrados coordenadas $\left(E, \pi, X, F, K, U_{\alpha}, \varphi_{\alpha}\right)$ $e\left(E, \pi, X, F, K, U_{\mu}^{\prime}, \varphi_{\mu}^{\prime}\right)$ que têm em comum $E, \pi, X, F$ eK são chamados equivalentes no sentido estrito $s e$

$$
\bar{\Theta}_{\mu \alpha}(x)=\varphi_{\mu, x}^{\prime} \cdot \varphi_{\alpha, x}^{-1} \in K \quad \text { para } \quad x \in U_{\alpha} \cap U_{\mu}^{\prime}
$$




$$
\bar{\Theta}_{\mu \alpha}: U_{\alpha} \cap U_{\mu}^{\prime} \rightarrow K \quad \text { é contínua. }
$$

Uma classe de equivalência $\xi=(E, \pi, X, F, K)$ de fibrados coordenados com respeito a relação de equivalência acima é chamada de um fibrado, e E é chamado de espaço total, $\pi$ de projeção, $X$ de espaço base, $F$ de fibra, $e K$ de grupo estrutural. Em um fibrado coordenadas $\left(E, \pi, X, F, K, U_{\alpha}, \varphi_{\alpha}\right)$ chamamos $U_{\alpha}$ de vizinhança coordenada, $\varphi_{\alpha}$ de função coordenada, $e \Theta_{\beta \alpha}$ de função de transição. Também, para cada $x \in X$, denotamos por $E_{x}=\pi^{-1}(x)$ a fibra sobre $x$.

Um fibrado coordenadas que é um representante de um fibrado $\xi$ é freqüentemente chamado, por simplicidade, de fibrado e abreviamos a notação de um fibrado $\xi=(E, \pi, X, F, K)$ para $\pi: E \rightarrow X$ ou E.

Um fibrado em que $E=X \times F$ e id $: E \rightarrow X \times F$ é uma função coordenada é chamado de fibrado produto.

Definição 1.4.3. Sejam $\xi=(E, \pi, X, F, K) \quad$ e $\xi^{\prime}=\left(E^{\prime}, \pi^{\prime}, X^{\prime}, F, K\right)$ dois fibrados tendo a mesma fibra e o mesmo grupo estrutural. Uma aplicação contínua $\bar{f}: E \rightarrow E^{\prime}$ é chamada de aplicação fibrada de $\xi$ em $\xi^{\prime}$ se as duas condições seguintes são satisfeitas:

1. Existe uma aplicação contínua $f: X \rightarrow X^{\prime}$ tal que o diagrama seguinte comuta

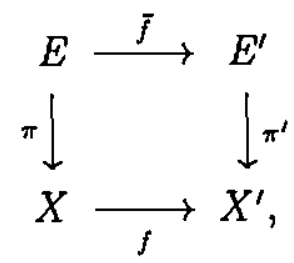

2. Sejam $\left\{U_{\alpha}, \varphi_{\alpha}\right\}$ e $\left\{V_{\mu}^{\prime}, \varphi_{\mu}^{\prime}\right\}$ pares de vizinhanças coordenadas e funções coordenadas de $\xi$ e $\xi^{\prime}$ respectivamente. Para todos $\alpha, \mu \operatorname{com} U_{\alpha} \cap f^{-1}\left(V_{\mu}^{\prime}\right) \neq \emptyset$ denotamos

$$
f_{\mu \alpha}(x)=\varphi_{\mu, f(x)}^{\prime} \cdot \bar{f} \cdot \varphi_{\alpha, x}^{-1} \quad \text { para } x \in U_{\alpha} \cap f^{-1}\left(V_{\mu}^{\prime}\right) .
$$


Então

$$
f_{\mu \alpha}(x) \in K \subset \operatorname{Homeo}(F)
$$

$e$

$$
f_{\mu \alpha}: U_{\alpha} \cap f^{-1}\left(V_{\mu}^{\prime}\right) \rightarrow K \quad \text { é contínua. }
$$

Se $X=X^{\prime}$ e $f=i d$ no acima, então $\bar{f}$ é chamado de um isomorfismo de fibrados. Quando existe um isomorfismo entre dois fibrados $\xi$ e $\xi^{\prime}$ dizemos que $\xi$ e $\xi^{\prime}$ são fibrados isomorfos (ou equivalentes) e denotamos $\xi \cong \xi^{\prime}$ ou $E \cong E^{\prime}$.

Um fibrado que é isomorfo ao fibrado produto é chamado de fibrado trivial.

Um fibrado $(E, \pi, X, F, K)$ em que $F=K$ e $K$ opera por translação à esquerda é chamado de fibrado principal, ou $K$-fibrado principal, e é denotado por $(P, \pi, X, K)$

Proposição 1.4.4. Para um fibrado principal $\eta=(P, \pi, X, K)$ existe uma $K$ ação à direita canonicamente livre sobre $P$ tal que a fibra $\pi^{-1}(x)$ é $K$-invariante. Mais ainda, a projeção $\pi: P \rightarrow X$ induz um homeomorfismo $\bar{\pi}: \frac{P}{K} \rightarrow X$.

Se $X$ é um $G$-espaço à direita e Y é um $G$-espaço à esquerda, podemos considerar a ação diagonal de $G$ sobre $X \times Y$, isto é:

$$
G \times(X \times Y) \rightarrow X \times Y \quad \text { dada por } \quad(g,(x, y)) \rightarrow g \cdot(x, y)=\left(x g^{-1}, g y\right) .
$$

O espaço das órbitas desta ação é chamado de produto torcido de $X$ e $Y$, e é denotado por

$$
X \times_{G} Y=\frac{X \times Y}{G} .
$$

Definição 1.4.5. Dados um K-fibrado principal $\pi: P \rightarrow X$ e FumK-espaço efetivo podemos construir um fibrado com fibra $F$ e grupo estrutural $K$ que é chamado de fibrado associado ao K-fibrado principal $P$, isto é:

$$
p: P \times_{K} F \rightarrow X \quad \text { definida por }[z, y] \rightarrow p([z, y])=\pi(z) .
$$


Teorema 1.4.6. Dois fibrados $E$ e $E^{\prime}$ tendo os mesmos espaço base, fibra $e$ grupo estrutural são isomorfos se e só se seus fibrados principais associados são isomorfos.

Definição 1.4.7. Uma secção para um fibrado $\pi: E \rightarrow B$ é uma aplicação continua $s: B \rightarrow E$ tal que $\pi . s=i d_{B}$.

Teorema 1.4.8. Seja $\pi: P \rightarrow X$ um fibrado principal. Então $P$ é trivial se $e$ só se $\pi$ tem uma secção.

Corolário 1.4.9. Um fibrado é trivial se e só se o fibrado principal associado admite uma seç̧ão.

Definição 1.4.10. Dado um fibrado $\xi=(E, \pi, X, F, K)$ e uma aplicação contínua $f: Y \rightarrow X$, obtemos um fibrado com fibra $F$, grupo estrutural $K$ e espaço base $Y$, chamado de fibrado induzido e denotado por $f ! \xi$, considerando

$$
f^{\prime} \xi=\{(y, z) \in Y \times E \mid f(y)=\pi(z)\}
$$

e o diagrama comutativo

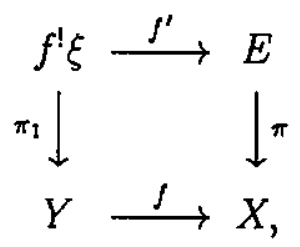

onde $f^{\prime}$ é uma aplicação fibrada e $\pi_{1}$ é a projeção no primeiro fator.

Definição 1.4.11. Sejam $E$ e $X$ espaços topológicos $e \pi: E \rightarrow X$ uma aplicação contínua. Se as condições 1 e 2 seguintes são satisfeitas, então $(E, \pi, X)$ é chamado de fibrado vetorial. 
1. Para todo $x, \pi^{-1}(x)$ tem a estrutura de um espaço vetorial sobre os números reais $\mathbb{R}$.

2. Para todo $x$, existe uma vizinhança aberta $U$ de $x$ e um homeomorfismo $\varphi: \pi^{-1}(U) \rightarrow U \times \mathbb{R}^{n}$ tal que $\quad p . \varphi=\pi \quad$ e, para cada $y \in U$

$$
\varphi_{\mid \pi^{-1}(y)}: \pi^{-1}(y) \rightarrow\{y\} \times \mathbb{R}^{n} \equiv \mathbb{R}^{n} \text { é um isomorfismo linear }
$$

onde $p: U \times \mathbb{R}^{n} \rightarrow U$ denota a projeção.

Quando a fibra $F$ de um fibrado vetorial $\pi: E \rightarrow X$ tem dimensão constante, isto é, $F$ é um espaço vetorial $n$-dimensional, dizemos que $\pi: E \rightarrow X$ é um fibrado vetorial $\boldsymbol{n}$-dimensional. Assim, um fibrado vetorial $n$-dimensional pode ser identificado com um fibrado com fibra $\mathbb{R}^{n}$ e grupo estrutural $G L(n, \mathbb{R})$ (às vezes chamado também de $\boldsymbol{n}$-plano fibrado ou $\boldsymbol{R}^{\boldsymbol{n}}$-fibrado).

Em particular, denotaremos o fibrado vetorial $\boldsymbol{n}$-dimensional trivial por $\varepsilon^{n}$ ou simplesmente por $n$, isto é,

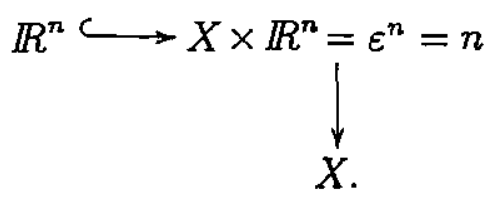

Denotaremos o fibrado linha canônico sobre $\boldsymbol{R} \boldsymbol{P}^{\boldsymbol{n}}$, espaço projetivo real $n$-dimensional, por $\gamma_{n}^{1}=\left(E\left(\gamma_{n}^{1}\right), \pi_{1}, \mathbb{R} P^{n}\right)$, onde

$$
E\left(\gamma_{n}^{1}\right)=\left\{(\{ \pm x\}, v) \in \mathbb{R} P^{n} \times \mathbb{R}^{n+1} ; v \text { é múltiplo de } x\right\}
$$

e

$$
\pi_{1}: E\left(\gamma_{n}^{1}\right) \rightarrow \mathbb{R} P^{n} \text { é a projeção no primeiro fator. }
$$

Definição 1.4.12. Dada uma secção $s: B(\xi) \rightarrow E(\xi)$ de um fibrado vetorial $\xi$, dizemos que s é uma secção totalmente não-nula se $s(b)$ é um vetor não-nulo de $F_{b}(\xi)$ para cada $b \in B(\xi)$. 
Definição 1.4.13. Dada uma coleção de seç̧ões $s_{1}, \ldots, s_{n}$ de um fibrado vetorial $\xi$, dizemos que $s_{1}, \ldots, s_{n}$ são secções totalmente independentes se para cada $b \in B(\xi)$ os vetores $s_{1}(b), \ldots, s_{n}(b)$ são linearmente independentes.

Teorema 1.4.14. Um fibrado vetorial $n$-dimensional $\xi$ é trivial se e só se $\xi$ admite $n$ secções $s_{1}, \ldots, s_{n}$ que são totalmente independentes.

Definição 1.4.15. Sejam $G$ um grupo topológico e $\pi: E \rightarrow X$ um fibrado vetorial tal que $E$ e X são G-espaços e $\pi$ é uma G-aplicação. Se para todo $g \in G e$ $x \in X, a$ aplicação

$$
g: \pi^{-1}(x) \rightarrow \pi^{-1}(g x)
$$

é um isomorfismo linear, então $\pi: E \rightarrow X$ é chamado de um $G$-fibrado vetorial.

Resumiremos agora algumas construções básicas envolvendo fibrados.

Sejam $\pi: E \rightarrow X$ e $\pi^{\prime}: E^{\prime} \rightarrow X^{\prime}$ dois $G$-fibrados vetoriais. O fibrado produto de $\mathrm{E}$ e $\mathrm{E}$ ' é definido como o produto cartesiano

$$
\pi \times \pi^{\prime}: E \times E^{\prime} \rightarrow X \times X^{\prime}
$$

Quando $X=X^{\prime}$ a soma de Whitney de E e E' é denotada por $E \oplus E^{\prime}$ e é definida como o fibrado induzido por $E \times E^{\prime}$ e pela aplicação diagonal $d: X \rightarrow X \times X$ (dada por $d(x)=(x, x)$ ), isto é,

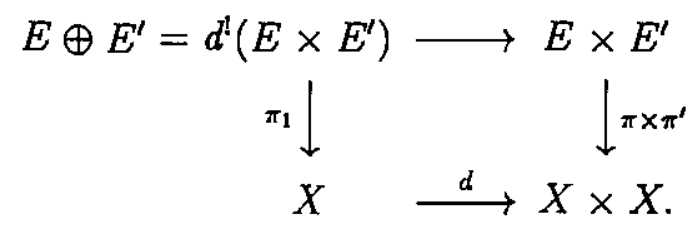

Seja $\xi$ um fibrado vetorial $F \rightarrow E \stackrel{\pi}{\rightarrow} B$ e $\bar{B} \subset B$ um subconjunto. Denotamos por $\xi_{\left.\right|_{B}}$ o fibrado restrição de $\xi$ a $\bar{B}$, isto é, 


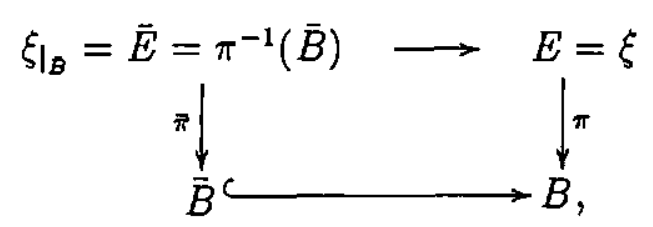

onde $\bar{\pi}$ é a restrição de $\pi$ a $\bar{E}$. Observe que a fibra é a mesma nos dois fibrados.

Lema 1.4.16. Se $\bar{f}: E \rightarrow E^{\prime} \quad$ é uma aplicação fibrada e $f: B \rightarrow B^{\prime}$ é a aplicação correspondente dos espaços base, então $E \cong f^{\prime} E^{\prime}$.

Esquematicamente,

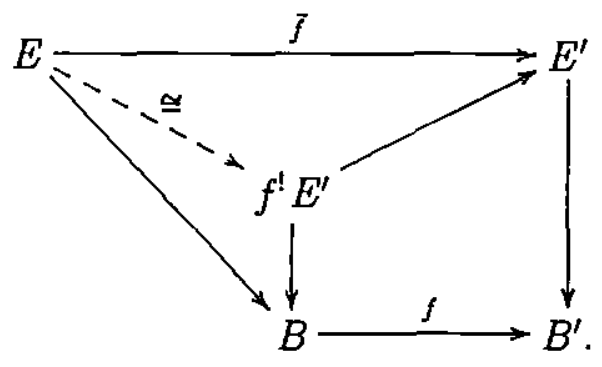

Sejam $\xi$ e $\eta$ fibrados vetoriais sobre o mesmo espaço base $B$ com $E(\xi) \subset E(\eta)$. Dizemos que $\xi$ é um subfibrado de $\eta$ se cada fibra $F_{b}(\xi)$ é um sub-espaço vetorial da fibra correspondente a $F_{b}(\eta)$.

Lema 1.4.17. Se $\xi_{1}$ e $\xi_{2}$ são subfibrados vetoriais de $\eta: E \rightarrow B$ tais que $F_{b}(\eta)=F_{b}\left(\xi_{1}\right) \oplus F_{b}\left(\xi_{2}\right), \forall b \in B, \quad$ então $\eta \cong \xi_{1} \oplus \xi_{2}$.

Teorema 1.4.18. Dado um subfibrado $\xi \subset \eta$, onde $\eta$ é um fibrado vetorial dotado de uma métrica Euclidiana, existe um subfibrado $\xi^{\perp} \subset \eta$ tal que $\eta \cong \xi \oplus \xi^{\perp}$.

O fibrado $\xi^{\perp}$ acima é chamado de complemento ortogonal de $\xi$ em $\eta$.

Quando uma variedade diferenciável $N$ admite uma métrica Euclideana dizemos que $N$ é uma variedade Riemanniana. 
Considerando uma imersão $f: M \rightarrow N$ entre variedades diferenciáveis, com $N$ Riemanniana, temos $\tau_{M}$ subfibrado do fibrado induzido $f^{!} \tau_{N}$, isto é,

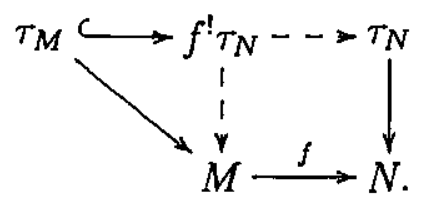

Daí, usando o Lema 1.4.16, temos

Corolário 1.4.19. Se $f: M \rightarrow N$ é uma imersão entre variedades diferenciáveis, com $N$ Riemanniana, então existe uma decomposição em soma de Whitney

$$
f^{\prime} \tau_{N} \cong \tau_{M} \oplus \nu_{f}
$$

O fibrado $\nu_{f}$ é chamado de fibrado normal da imersão $f$.

Em particular, quando $f=i$, a inclusão, temos

$$
i^{!} \tau_{N} \cong \tau_{N_{\mid M}}=\tau_{M} \oplus \tau_{M}^{\perp} .
$$




\subsection{Fibrado Universal (Espaços Classificantes)}

Dado um grupo de Lie compacto $G$, existe (Construção de Milnor) um $G$ fibrado principal $\pi: E(G) \rightarrow B(G)$, onde $E(G)$ é contrátil $\left(\pi_{i}(E(G))=0, \forall i\right) \mathrm{e}$ $B(G)=\frac{E(G)}{G}$ é determinado de modo único a menos de homotopia.

Se $\quad p: P \rightarrow X$ é um $G$-fibrado principal com base paracompacta $X$, então existe uma aplicação $f: X \rightarrow B(G)$, tal que o fibrado induzido $f^{\prime}(E(G))$ é isomorfo a $P$.

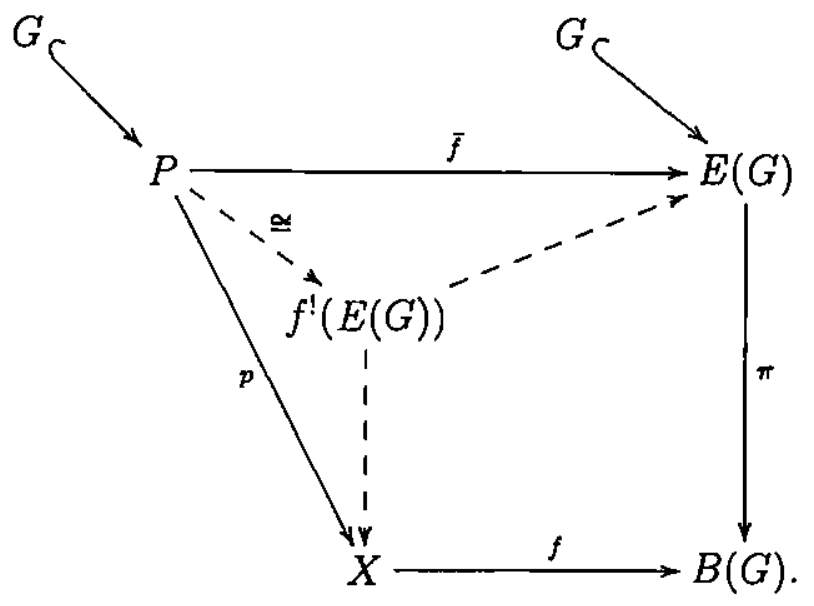

Desta propriedade, o fibrado $\pi: E(G) \rightarrow B(G)$ é chamado de $G$-fibrado principal universal, o espaço $B(G)$ de espaço classificante de $G$ e a aplicação $f$ de aplicação classificante de $P$. Ver, por exemplo, [12]. 


\section{Capítulo 2}

\section{Bordismo e Números de}

\section{Stiefel-Whitney}

Neste capítulo reescrevemos alguns resultados sobre bordismo e números de Stiefel-Whitney visando basicamente chegar ao Teorema de Conner-Floyd 2.5.2 que tem um papel fundamental no capítulo seguinte. As provas destes resultados podem ser encontradas em [11] e [4]. Na última seção obtemos uma condição para que uma $\mathbb{Z}_{2}$-variedade tendo conjunto de pontos fixos 1-codimensional tenha classse de bordismo nula. Consideraremos daqui em diante apenas variedades diferenciáveis compactas e diremos simplesmente variedades.

\subsection{Bordismo}

Definição 2.1.1. Uma n-variedade $M$ borda se existe uma $(n+1)$-variedade (diferenciável compacta) $W$ com $\partial W=M$. Duas $n$-variedades $M$ e $N$ são bordantes, denotamos $M \sim N$, se a sua união disjunta $M \dot{U} N$ borda, isto é,

$$
M \sim N \Leftrightarrow \exists W^{n+1} \text { com } \partial W=M \dot{U} N .
$$

A relação "ser bordante" é uma relação de equivalência e denotamos a classe de equivalência de uma variedade $M$ por $[M]_{2}$. 
Definição 2.1.2. O conjunto das classes de equivalência da relação acima é chamádo de grupo de bordismo não-orientado de dimensão $n$ e é denotado por

$$
\mathcal{N}_{n}=\left\{[M]_{2} ; M^{n} \text { é variedade diferenciável compacta }\right\}
$$

O conjunto $\mathcal{N}_{n}$ é um grupo com a operação

$$
[M]_{2}+[N]_{2}=[M \dot{\cup} N]_{2}
$$

Mais ainda, $\mathcal{N}_{*}=\sum \mathcal{N}_{n}$ é um anel com a multiplicação induzida pelo produto cartesiano.

Thom em [13] mostrou que:

Teorema 2.1.3. $\mathcal{N}_{*}$ é uma álgebra polinomial sobre $\mathbb{Z}_{2}$ com um gerador em cada dimensão não da forma $2^{j}-1$.

Explicitamente, por [3, Teorema 13.17],

$$
\mathcal{N}_{*}=\mathbb{Z}_{2}\left[X_{2}, X_{4}, X_{5}, X_{6}, X_{8}, X_{9}, \ldots\right], \text { onde } X_{2 i}=\left[\mathbb{R R} P^{2 i}\right]_{2} \text {. }
$$

Fixemos agora um espaço $X$. Consideremos o conjunto os pares $(M, f)$, onde $M$ é uma n-variedade e $f: M \rightarrow X$ é uma aplicação (diferenciável).

Definição 2.1.4. Dizemos que um par $(M, f)$ borda se existe uma $(n+1)$-variedade (diferenciável compacta) $W$ e uma aplicação (diferenciável) $F: W \rightarrow X$ tal que $\partial W=M$ e $F_{\left.\right|_{M}}=f$. Dois pares $(M, f) e(N, g)$ são bordantes, denotamos $(M, f) \sim(N, g)$, se a sua união disjunta $(M \dot{\cup} N, f \dot{\cup} g)$ borda, isto é,

$$
(M, f) \sim(N, g) \Leftrightarrow\left\{\begin{array}{l}
\exists W^{n+1} \mid \quad \partial W=M \dot{\cup} N \\
\exists F: W \rightarrow X \mid \quad F_{\left.\right|_{M}}=f \text { e } F_{\left.\right|_{N}}=g .
\end{array}\right.
$$

Esquematicamente, 


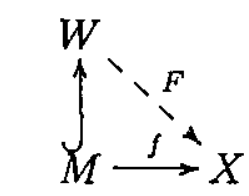

$\cdot(M, f)$ borda

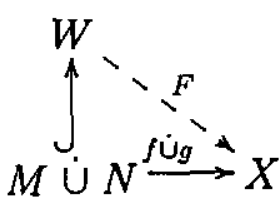

$(M, f)$ e $(N, g)$ bordantes

A relação acima também é uma relação de equivalência e denotamos a classe de equivalência de um par $(M, f)$ por $[M, f]_{2}$.

Definição 2.1.5. O conjunto das classes de equivalência da relação acima é chamado de grupo de bordismo não-orientado de dimensão $n$ do espaço $X$ $e$ é denotado por

$$
\mathcal{N}_{n}(X)=\left\{[M, f]_{2} ; M^{n} \text { variedade e } f: M \rightarrow X \text { aplicação }\right\}
$$

O conjunto $\mathcal{N}_{n}(X)$ é um grupo com a operação

$$
[M, f]_{2}+[N, g]_{2}=[M \dot{U} N, f \dot{U} g]_{2},
$$

onde $(f \dot{U} g)_{\left.\right|_{M}}=f$ e $(f \dot{U} g)_{\left.\right|_{N}}=g$.

Em particular, quando $X$ é um ponto temos $\mathcal{N}_{n}(X)=\mathcal{N}_{n}$.

Obs.: Conner \& Floyd em [4] consideram mais geralmente grupos de bordismo de um $\operatorname{par}(X, A)$.

Existe também uma teoria de bordismo equivariante para $G$-variedades, onde $G$ é um grupo finito.

Denotaremos aqui uma $G$-variedade $M^{n}$ por $\left(G, M^{n}\right)$.

Diremos que $\left(G, M^{n}\right)$ é uma $G$-variedade principal quando a ação de $G$ em $M^{n}$ é livre.

Quando $\left(G, M_{1}^{n}\right)$ e $\left(G, M_{2}^{n}\right)$ são $G$-variedades equivalentes (ver após a Defi- 
nição 1.1.2) denotamos simplesmente

$$
\left(G, M_{1}^{n}\right)=\left(G, M_{2}^{n}\right)
$$

Definição 2.1.6. Seja $\left(G, M^{n}\right)$ uma $G$-variedade principal fechada. Dizemos que $\left(G, M^{n}\right)$ borda equivariantemente se existe uma G-variedade principal compacta $\left(G, W^{n+1}\right)$ com $\left(G, \partial W^{n+1}\right)=\left(G, M^{n}\right)$. Duas $G$-variedades principais $\left(G, M_{1}^{n}\right)$ e $\left(G, M_{2}^{n}\right)$ são bordantes equivariantemente se a união disjunta $\left(G, M_{1}^{n} \cup M_{2}^{n}\right)$ borda equivariantemente.

A relação acima é uma relação de equivalência e denotamos a classe de equivalência de uma variedade $\left(G, M^{n}\right)$ por $\left[G, M^{n}\right]_{2}$.

Definição 2.1.7. O conjunto das classes de equivalência da relação acima é chamado de grupo de bordismo equivariante não-orientado de dimensão $n$ do grupo $G$ e é denotado por $\mathcal{N}_{n}(G)$.

O conjunto $\mathcal{N}_{n}(G)$ é um grupo abeliano com a operação soma definida por

$$
\left[G, M_{1}^{n}\right]_{2}+\left[G, M_{2}^{n}\right]_{2}=\left[G, M_{1}^{n} \dot{\cup} M_{2}^{n}\right]_{2} \text {. }
$$

A soma direta fraca $\mathcal{N}_{*}(G)=\sum_{n=0}^{\infty} \mathcal{N}_{n}(G)$ torna-se um $\mathcal{N}_{*}$-módulo (à direita) graduado ao considerar a operação

$$
\left[G, M^{n}\right]_{2} \cdot\left[V^{m}\right]_{2}=\left[G, M^{n} \times V^{m}\right]_{2},
$$

onde $[G, M]_{2} \in \mathcal{N}_{*}(G)$ e $\left[V^{m}\right]_{2} \in \mathcal{N}_{*}$, sendo a ação em $M^{n} \times V^{m}$ dada por $g(x, y)=(g x, y)$.

Chamamos $\mathcal{N}_{*}(G)$ de módulo de bordismo do grupo finito $G$.

Obs.: Conner \& Floyd em [4] consideram mais geralmente $\mathcal{N}_{*}(G, A)$ onde as ações livres são trocadas por ações $A$-livres. 


\subsection{Classes de Stiefel-Whitney}

Originalmente considerava-se classes de Stiefel de uma variedade, classes de Whitney de um fibrado vetorial e classes de Stiefel-Whitney do fibrado tangente à uma variedade. Posteriormente estes conceitos foram unificados, considerando classes de Stiefel-Whitney de um fibrado vetorial. Nesta seção resumimos a definição explícita das classes de Stiefel-Whitney de um fibrado vetorial, como em Conner-Floyd [4]. Posteriormente definimos axiomaticamente as classes de Stiefel-Whitney e resumimos algumas de suas propriedades, seguindo Milnor [11].

Dado um grupo de Lie $G$, consideremos o $G$-fibrado universal

$$
\pi: E(G) \rightarrow B(G)
$$

Para $G=\mathbb{Z}_{2}$ temos $B\left(\mathbb{Z}_{2}\right)=\mathbb{R} P^{\infty}$, onde $I R P^{\infty}$ é a união ascendente $I R P^{1} \subset \mathbb{I R} P^{2} \subset \cdots \subset \mathbb{I R} P^{n} \subset \ldots$ de espaços projetivos.

Como $H^{i}\left(I R P^{\infty} ; \mathbb{Z}_{2}\right)=\mathbb{Z}_{2}, \forall i$, temos $H^{*}\left(I R P^{\infty} ; \mathbb{Z}_{2}\right)=\mathbb{Z}_{2}[t]$, uma álgebra polinomial com um gerador 1-dimensional $t \in H^{1}\left(I R P^{\infty} ; \mathbb{Z}_{2}\right)$.

$$
\begin{aligned}
& \text { Para } G=\left(\mathbb{Z}_{2}\right)^{n}, \text { como } B\left(G_{1} \times G_{2}\right)=B\left(G_{1}\right) \times B\left(G_{2}\right), \text { temos } \\
& H^{*}\left(B\left(\left(\mathbb{Z}_{2}\right)^{n}\right) ; \mathbb{Z}_{2}\right)=\mathbb{Z}_{2}\left[t_{1}, \ldots, t_{n}\right]
\end{aligned}
$$

com cada $t_{i}$ 1-dimensional.

Para $G=O(n)$, o grupo ortogonal, denotando por $D$ o subgrupo das matrizes ortogonais diagonais em $O(n)$, temos $D \cong\left(\mathbb{Z}_{2}\right)^{n}$ e o seguinte resultado: A inclusão $\left(\mathbb{Z}_{2}\right)^{n} \subset O(n)$ induz um monomorfismo

$$
\rho: H^{*}\left(B(O(n)) ; \mathbb{Z}_{2}\right) \rightarrow H^{*}\left(B\left(\left(\mathbb{Z}_{2}\right)^{n}\right) ; \mathbb{Z}_{2}\right)=\mathbb{Z}_{2}\left[t_{1} \ldots, t_{n}\right]
$$

cuja imagem são os polinômios simétricos em $t_{1}, \ldots, t_{n}$. 
Em particular, denotaremos os polinômios simétricos elementares

$$
\sum_{j_{1}<\cdots<j_{k}} t_{j_{1}} \ldots t_{j_{k}}
$$

simplesmente por $\sum t_{1} \ldots t_{k}$.

Definição 2.2.1. As classes de Stiefel-Whitney universais do fibrado $\pi: E(O(n)) \rightarrow B(O(n))$ são as classes de cohomologia $w_{i} \in H^{i}\left(B(O(n)) ; \mathbb{Z}_{2}\right)$, $1 \leq i \leq n$, tais que $\rho\left(w_{i}\right)=\sum t_{1} \ldots t_{i}$, os polinômios simétricos elementares. $A$ classe de Stiefel-Whitney universal total de $E(O(n)) e ́$

$$
w=1+w_{1}+\cdots+w_{n}
$$

Temos então que

$$
H^{*}\left(B(O(n)) ; \mathbb{Z}_{2}\right)=\mathbb{Z}_{2}\left[w_{1}, \ldots, w_{n}\right]
$$

Considere agora $\tau: E \rightarrow X$ um fibrado vetorial $n$-dimensional $\operatorname{com} X$ um $\mathrm{CW}$-complexo. Tomando um produto interno sobre o fibrado, podemos considerar $\tau$ como um $O(n)$-fibrado. Assim existe uma aplicação fibrada

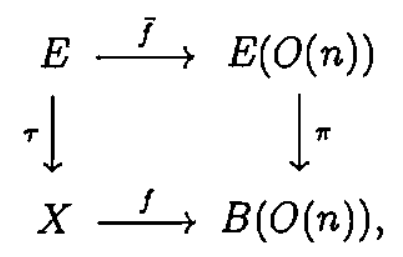

onde $\pi: E(O(n)) \rightarrow B(O(n))$ é um $O(n)$-fibrado universal com fibra $\mathbb{R}^{n}$. Por universalidade, o homomorfismo

$$
f^{*}: H^{*}\left(B(O(n)) ; \mathbb{Z}_{2}\right) \rightarrow H^{*}\left(X ; \mathbb{Z}_{2}\right)
$$

é independente da aplicação fibrada particular. 
Definição 2.2.2. As classes de Stiefel-Whitney de um $O(n)$-fibrado vetorial $\tau: E \rightarrow X$ são definidas como

$$
w_{i}(\tau)=f^{*}\left(w_{i}\right) \in H^{i}\left(X ; \mathbb{Z}_{2}\right),
$$

onde os $w_{i}$ 's são as classes de Stiefel-Whitney universais.

Agora vejamos a definição axiomática das classes de Stiefel-Whitney.

As classes de cohomologia de Stiefel-Whitney de um fibrado vetorial são definidas através de quatro axiomas:

Axioma 2.2.3. Para cada fibrado vetorial $\xi$ corresponde uma seqüência de classes de cohomologia

$$
w_{i}(\xi) \in H^{i}\left(B(\xi) ; \mathbb{Z}_{2}\right), \quad i=0,1,2, \ldots,
$$

chamadas de classes de Stiefel-Whitney de $\xi$. Se $\xi$ é um fibrado n-dimensional então

$$
\begin{aligned}
& w_{0}(\xi)=1 \in H^{0}\left(B(\xi) ; \mathbb{Z}_{2}\right), \quad \text { elemento unidade, } \\
& w_{i}(\xi)=0 \text { para } i>n, \quad \text { a classe nula. }
\end{aligned}
$$

Axioma 2.2.4 (Naturalidade). Se $f: B(\xi) \rightarrow B(\eta)$ é coberta por uma aplicação fibrada de $\xi$ em $\eta$ então

$$
w_{i}(\xi)=f^{*} w_{i}(\eta)
$$

onde

$$
f^{*}: H^{i}\left(B(\eta) ; \mathbb{Z}_{2}\right) \rightarrow H^{i}\left(B(\xi) ; \mathbb{Z}_{2}\right)
$$

Axioma 2.2.5 (Teorema do Produto de Whitney). Se $\xi$ e $\eta$ são fibrados vetoriais sobre o mesmo espaço base, então

$$
w_{k}(\xi \oplus \eta)=\sum_{i=0}^{k} w_{i}(\xi) \smile w_{k-i}(\eta)
$$

onde $\smile$ é o produto cup. 
Axioma 2.2.6. Para o fibrado linha canônico $\gamma_{1}^{1}$ sobre a esfera $S^{1}$, a classe de Stiefel-Whitney $w_{1}\left(\gamma_{1}^{1}\right)$ é não-nula.

As classes de Știefel-Whitney da primeira definição satisfazem os quatro axiomas acima.

Milnor [11] prova a existência de classes de Stiefel-Whitney dando uma construção em termos das "Operações Quadrado de Steenrod".

As classes de Stiefel-Whitney têm as seguintes propriedades.

Proposição 2.2.7. Se $\xi$ é isomorfo a $\eta$ então $w_{i}(\xi)=w_{i}(\eta)$.

Proposição 2.2.8. Se $\varepsilon$ é um fibrado vetorial trivial então $w_{i}(\varepsilon)=0$ para $i>0$.

Proposição 2.2.9. Se $\varepsilon$ é trivial então $w_{i}(\varepsilon \oplus \eta)=w_{i}(\eta)$.

Proposição 2.2.10. Se $\xi$ é um fibrado n-dimensional com uma métrica Euclideana que possui uma seç̧ão totalmente não-nula, então $w_{n}(\xi)=0$. Se $\xi$ possui $k$ seç̧ões que são totalmente independentes, então

$$
w_{n-k+1}(\xi)=w_{n-k+2}(\xi)=\cdots=w_{n}(\xi)=0 .
$$

Denotemos por $H^{*}\left(B ; \mathbb{Z}_{2}\right)$ o anel consistindo de todas séries formais infinitas

$$
a=a_{0}+a_{1}+a_{2}+\cdots, \quad \text { com } a_{i} \in H^{i}\left(B, \mathbb{Z}_{2}\right) .
$$

A operação produto deste anel é dada pela fórmula

$$
\begin{aligned}
a . b & =\left(a_{0}+a_{1}+a_{2}+\cdots\right)\left(b_{0}+b_{1}+b_{2}+\cdots\right) \\
& =\left(a_{0} b_{0}\right)+\left(a_{1} b_{0}+a_{0} b_{1}\right)+\left(a_{2} b_{0}+a_{1} b_{1}+a_{0} b_{2}\right)+\cdots .
\end{aligned}
$$


Definição 2.2.11. A classe de Stiefel-Whitney total de um fibrado n-dimensional $\xi$ sobre $B$ é definida como o elemento

$$
w(\xi)=1+w_{1}+w_{2}+\cdots+w_{n}+0+\cdots
$$

do anel $H^{*}\left(B ; \mathbb{Z}_{2}\right)$.

O Teorema do Produto de Whitney torna-se então

$$
w(\xi \oplus \eta)=w(\xi) \cdot w(\eta)
$$

onde o lado direito é a operação produto do anel.

Quando $\xi=\tau_{M}$ denotamos por

$$
w(M)=w\left(\tau_{M}\right),
$$

a classe de Stiefel-Whitney total do fibrado tangente à variedade $M$ e diremos simplesmente classe de Stiefel-Whitney de $M$.

Como exemplo temos:

a) $w\left(S^{n}\right)=1$

b) $w\left(\mathbb{R} P^{n}\right)=(1+a)^{n+1}$, onde $a \in H^{1}\left(\mathbb{R} P^{n} ; \mathbb{Z}_{2}\right)=\mathbb{Z}_{2}$ é o gerador. 


\subsection{Números de Stiefel-Whitney}

Seja $M^{n}$ uma variedade diferenciável fechada, possivelmente desconexa. Denotamos a única classe de homologia fundamental de $M$ por

$$
[M] \in H_{n}\left(M ; \mathbb{Z}_{2}\right) .
$$

Para cada classe de cohomologia $v \in H^{n}\left(M ; \mathbb{Z}_{2}\right)$ denotamos o índice de Kronecker por

$$
v[M]=\left\langle v,[M]>\in \mathbb{Z}_{2} .\right.
$$

Seja $I=\left(i_{1}, \ldots, i_{r}\right)$ uma partição de $n$, isto é, $i_{1}, \ldots, i_{r}$ são inteiros não negativos com $i_{1}+\cdots+i_{r}=n$. Quando $I=n$ dizemos que $I$ é uma partição trivial de $n$. Para cada fibrado vetorial $\xi$ podemos formar o monômio

$$
w_{i_{1}}(\xi) \smile \cdots \smile w_{i_{r}}(\xi) \in H^{n}\left(B(\xi) ; \mathbb{Z}_{2}\right),
$$

onde $\smile$ denota o produto cap, que denotaremos simplesmente por

$$
w_{i_{1}}(\xi) \ldots w_{i_{r}}(\xi) .
$$

Em particular, considerando $\xi=\tau_{M}$, o fibrado tangente da variedade $M$, temos

$$
w_{i_{1}}\left(\tau_{M}\right) \ldots w_{i_{r}}\left(\tau_{M}\right) \in H^{n}\left(M ; \mathbb{Z}_{2}\right) .
$$

Definição 2.3.1. O número de Stiefel-Whitney da variedade fechada $M^{n}$, associado à partição $I=\left(i_{1}, \ldots, i_{r}\right)$ de $n$ é o inteiro mod 2

$$
W_{l}(M)=<w_{i_{1}}\left(\tau_{M}\right) \ldots w_{i_{r}}\left(\tau_{M}\right),[M]>.
$$

A importância dos números de Stiefel-Whitney é ilustrada pelo teorema seguinte e pela sua recíproca.

Teorema 2.3.2 (Pontrjagin). Se $B^{n+1}$ é uma variedade diferenciável compacta com $\partial B=M^{n}$, então todos os números de Stiefel-Whitney de $M$ são nulos. 
Como aplicação deste teorema temos: $\mathbb{R} P^{n}$ borda $\Leftrightarrow n$ é ímpar.

A recíproca do Teorema de Pontrjagin é devida a Thom.

Teorema 2.3.3 (Thom). Se todos os números de Stiefel-Whitney de $M$ são nulos, então existe uma variedade diferenciável compacta $B^{n+1}$ com $\partial B=M^{n}$.

Corolário 2.3.4. Duas n-variedades diferenciáveis fechadas $M_{1}$ e $M_{2}$ pertencem à mesma classe de bordismo não-orientado se e somente se todos os números de Stiefel-Whitney correspondentes de $M_{1}$ e $M_{2}$ são iguais.

A característica de Euler módulo 2 de uma variedade $M$ é um invariante da classe de bordismo pois considerando a partição trivial $I=n$ temos o seguinte relacionamento (ver [11, Corolário11.12]) entre o número de Stiefel-Whitney $W_{n}(M)$ e $\chi(M)$

Teorema 2.3.5. Se $M$ é uma variedade diferenciável compacta então

$$
W_{n}(M) \equiv \chi(M) \quad \bmod 2
$$




\subsection{Fibrado Involução}

Uma involução é um homeomorfismo $T: X \rightarrow X$ de período 2. Considerando $\mathbb{Z}_{2} \equiv\{1, T\}$, identificaremos uma involução sobre $X$ com uma ação de $\mathbb{Z}_{2}$ sobre $X$ e a denotaremos por $(T, X)$.

Como uma involução livre $\left(T, M^{n}\right)$ sobre uma variedade fechada é uma $\mathbb{Z}_{2^{-}}$ variedade principal fechada temos uma relação de bordismo e o grupo de bordismo $\mathcal{N}_{n}\left(\mathbb{Z}_{2}\right)$ (Ver Definição 2.1.7). Especificamente, $\left(T, M^{n}\right)$ borda, escrito $\left[T, M^{n}\right]_{2}=0$, se existe uma involução livre $\left(S, B^{n+1}\right)$ sobre uma variedade compacta com $\partial B^{n+1}=M^{n}$ e $S_{\left.\right|_{M^{n}}}=T$.

Consideremos então $\left(T, M^{n}\right)$ uma involução livre e $M \rightarrow \frac{M}{T}$ o $\mathbb{Z}_{2}$-fibrado principal associado. Por 1.5, existem: uma aplicação equivariante $M^{n} \rightarrow E\left(\mathbb{Z}_{2}\right)$, onde $E\left(\mathbb{Z}_{2}\right)$ é um $\mathbb{Z}_{2}$-fibrado universal, e uma aplicação induzida

$$
\rho: \frac{M^{n}}{T} \rightarrow \frac{E\left(\mathbb{Z}_{2}\right)}{T}=B\left(\mathbb{Z}_{2}\right)=\mathbb{R} P^{\infty}
$$

única a menos de homotopia, isto é, temos um diagrama

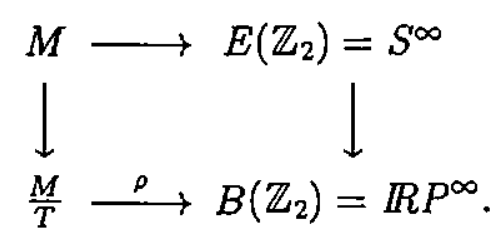

Temos então o diagrama induzido em cohomologia

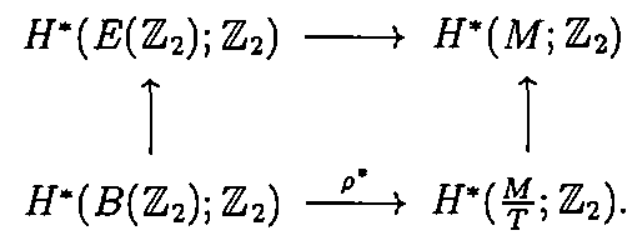

Como $H^{*}\left(B\left(\mathbb{Z}_{2}\right) ; \mathbb{Z}_{2}\right)=\mathbb{Z}_{2}[t]$, uma álgebra polinomial com um gerador 1-dimensional, podemos considerar, como na Definição 2.2.2,

$$
\rho^{*}(t) \in H^{1}\left(\frac{M}{T} ; \mathbb{Z}_{2}\right) .
$$


Denotaremos $c=\rho^{*}(t)$ e a chamaremos de a classe característica da involução $(T, M)$.

Sejam $\xi \rightarrow V^{n}$ um fibrado $k$-dimensional e $q: S(\xi) \rightarrow V^{n}$ o fibrado esfera associado a $\xi$, onde a fibra é uma $(k-1)$-esfera $S^{k-1}$ e $S(\xi)$ é uma $(n+k-1)$-variedade fechada.

Como a aplicação antipodal $A: S^{k-1} \rightarrow S^{k-1}$ está no centro de $O(k)$, temos correspondentemente uma involução livre preservando fibra sobre $S(\xi)$, que em cada fibra reduz-se à aplicaçâo antipodal e que denotaremos por $T: S(\xi) \rightarrow S(\xi)$.

Chamamos $(T, S(\xi))$ de o fibrado involuçāo associado a $\xi$.

Consideremos então, como acima, o $\mathbb{Z}_{2}$-fibrado principal $S(\xi) \rightarrow \frac{S(\xi)}{T}$ associado ao fibrado involução $(T, S(\xi))$, o diagrama correspondente

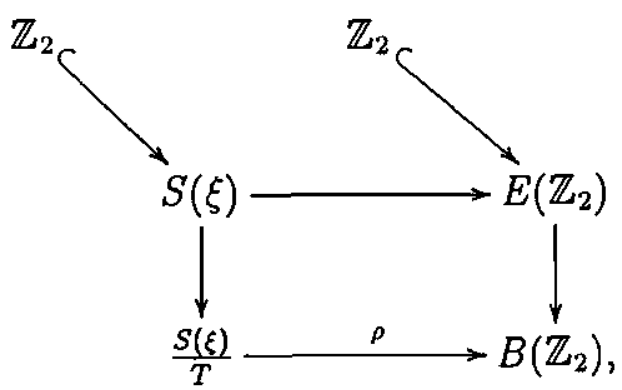

e $c=\rho^{*}(t)$ a classe característica da involução $(T, S(\xi))$.

Denotando $\mathbb{R} P(\xi)=\frac{S(\xi)}{T}$ temos também um diagrama comutativo

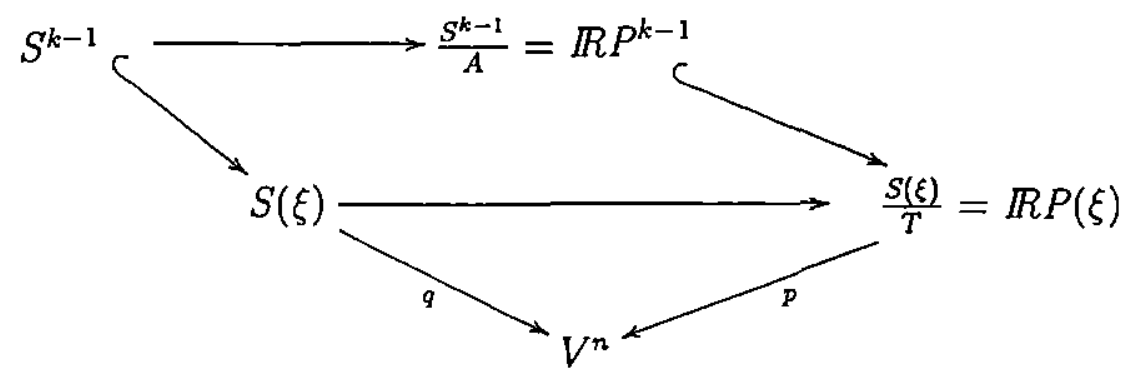

onde $p: \mathbb{R} P(\xi) \rightarrow V^{n}$ é um fibrado, com fibra $\mathbb{R} P^{k-1}$, chamado de fibrado espaço projetivo associado ao fibrado vetorial $\xi \rightarrow V^{n}$. 
Consideremos agora a inclusão $i: \mathbb{R} P^{k-1} \rightarrow \mathbb{R P}(\xi)$.

Por um lado, considerando os $\mathbb{Z}_{2}$-fibrados, temos o diagrama

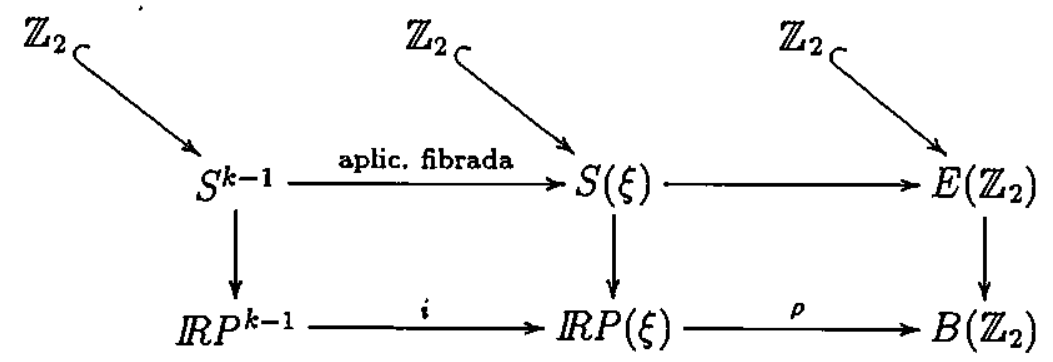

e correspondentemente

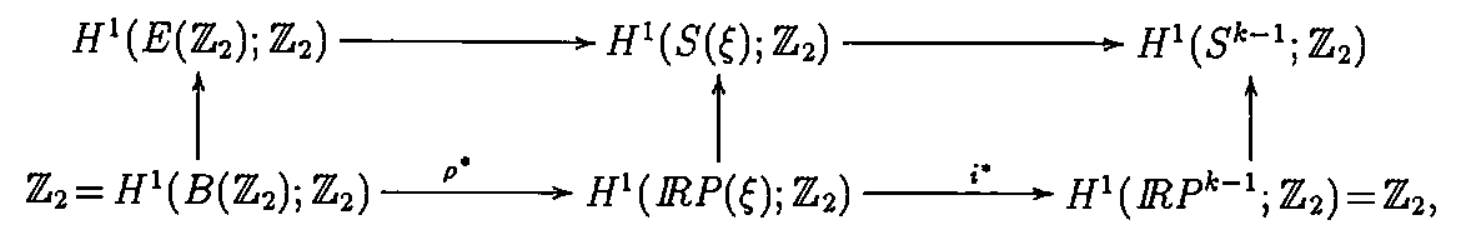

que associa

$$
t \longrightarrow \rho^{*}(t)=c \longrightarrow i^{*}(c)
$$

Assim, pela naturalidade das classes de Stiefel-Whitney, 2.2.4, a imagem $i^{*}(c)$ de $c \in H^{1}\left(\mathbb{R P}(\xi) ; \mathbb{Z}_{2}\right)$ pelo homomorfismo $i^{*}: H^{1}\left(\mathbb{R P}(\xi) ; \mathbb{Z}_{2}\right) \rightarrow$ $H^{1}\left(R P^{k-1} ; \mathbb{Z}_{2}\right)$ é o gerador de $H^{1}\left(\mathbb{R} P^{k-1} ; \mathbb{Z}_{2}\right)$.

Por outro lado, considerando $p: \mathbb{R P}(\xi) \rightarrow V^{n}$ e os seus fibrados tangentes, $\tau_{R P(\xi)}$ e $\tau_{V}$, temos

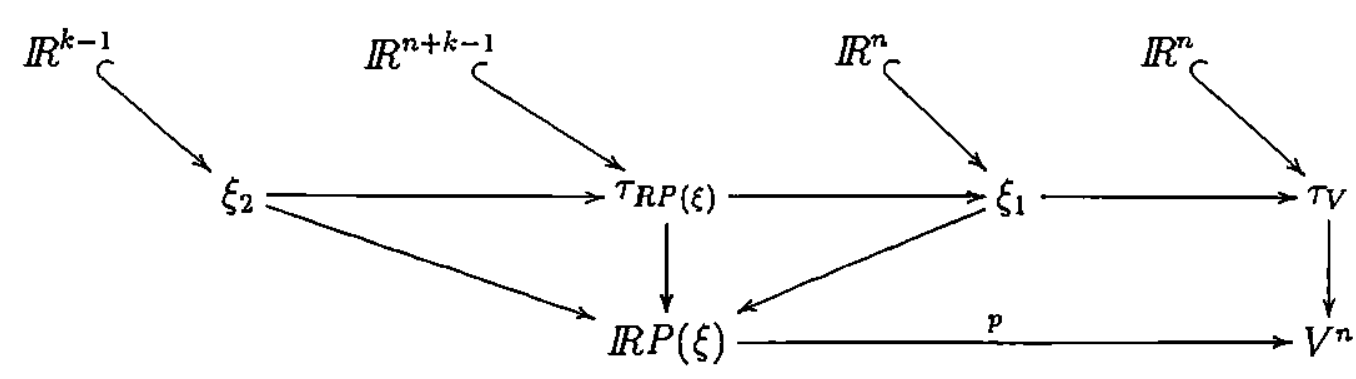


onde

$\xi_{1}=p^{!}\left(\tau_{V}\right)$ é o fibrado induzido por $p ;$

$\xi_{2}$ é o fibrado dos vetores de $\tau_{R P(\xi)}$ paralelos às fibras $\mathbb{R} P^{k-1}$ de $\mathbb{I R P}(\xi) \rightarrow V^{n}$.

Como $\operatorname{IRP}(\xi)$ é localmente um produto temos, usando o Lema 1.4.17,

$$
\tau_{R P(\xi)}=\xi_{1} \oplus \xi_{2} .
$$

(Observe que daí temos $\xi_{1 \mid R P^{k-1}} \cong \nu=\nu\left(\operatorname{IRP} P^{k-1}, \operatorname{IRP}(\xi)\right)$.)

Considerando $p: \mathbb{R P}(\xi) \rightarrow V^{n}$ temos por naturalidade que a classe de Stiefel-Whitney total de $\xi_{1}$ é dada por

$$
w\left(\xi_{1}\right)=p^{*}\left(w\left(V^{n}\right)\right)=p^{*}\left(\sum_{r=0}^{n} w_{r}\right)=\sum_{r=0}^{n} p^{*}\left(w_{r}\right)=1+p^{*}\left(w_{1}\right)+\cdots+p^{*}\left(w_{n}\right),
$$

onde $w_{r}=w_{r}\left(V^{n}\right), r=0,1,2, \ldots, n, \quad$ são as classes de Stiefel-Whitney de $\tau_{V^{n}}$.

A classe de Stiefel-Whitney total de $\xi_{2}$ ao longo da fibra em $\boldsymbol{I R P}(\boldsymbol{\xi})$ foi calculada por Borel-Hirzebruch [1].

Teorema 2.4.1 (Borel-Hirzebruch). A classe de Stiefel-Whitney total de $\xi_{2}$ ao longo da fibra em $\mathbb{R P}(\xi)$ é dada por

$$
w\left(\xi_{2}\right)=\sum_{s=0}^{k}(1+c)^{k-s} p^{*}\left(v_{s}\right)=(1+c)^{k}+(1+c)^{k-1} p^{*}\left(v_{1}\right)+\cdots+p^{*}\left(v_{k}\right),
$$

onde $v_{s}=w_{s}(\xi), s=1,2, \ldots, k, \quad$ são as classes de Stiefel-Whitney do fibrado vetorial $k$-dimensional $\xi \rightarrow V^{n}$.

Como $\xi_{2}$ é um fibrado $(k-1)$-dimensional segue também que vale a relação

$$
c^{k}=c^{k-1} p^{*}\left(v_{1}\right)+\cdots+p^{*}\left(v_{k}\right)
$$

Assim, a classe de Stiefel-Whitney total de $\boldsymbol{I R P}(\boldsymbol{\xi})$ é

$$
\begin{aligned}
w(\operatorname{IRP}(\xi)) & =w\left(\xi_{1} \oplus \xi_{2}\right)=w\left(\xi_{1}\right) \cdot w\left(\xi_{2}\right) \\
& =\left(\sum_{r=0}^{n} p^{*}\left(w_{r}\right)\right) \cdot\left(\sum_{s=0}^{k}(1+c)^{k-s} p^{*}\left(v_{p}\right)\right) .
\end{aligned}
$$


Explicitamente, cada classe de Stiefel-Whitney de $\operatorname{IRP}(\xi)$ é dada por

$$
w_{i}(\mathbb{R} P(\xi))=\sum_{r+s+q=i}\left(\begin{array}{c}
k-s \\
q
\end{array}\right) p^{*}\left(w_{r} v_{s}\right) c^{q}
$$

onde $\quad w_{r}=w_{r}\left(V^{n}\right) \in H^{r}\left(V^{n} ; \mathbb{Z}_{2}\right), \quad v_{s}=w_{s}\left(\xi \rightarrow V^{n}\right) \in H^{s}\left(V^{n} ; \mathbb{Z}_{2}\right)$ e $c \in$ $H^{1}\left(\operatorname{IRP}(\xi) ; \mathbb{Z}_{2}\right)$. 


\subsection{Classe de Bordismo de Variedade com Invo- lução}

Dada uma involução sobre uma variedade $M^{n}$ descrevemos sua classe de bordismo $[M]_{2} \in \mathcal{N}_{n}$ em termos de seu conjunto de pontos fixos.

Consideremos inicialmente o caso em que a involução $(T, M)$ é livre de pontos fixos.

Proposição 2.5.1. Se $M^{n}$ é uma variedade fechada admitindo uma involução livre de pontos fixos, então $M$ borda.

Uma prova geométrica deste fato é considerar

$T: M \rightarrow M$ a involução livre de pontos fixos,

$\theta: M \times I \rightarrow M \times I$ involução em $M \times I$ definida por $\theta(x, t)=(T x, 1-t)$.

Como $\theta$ é livre podemos considerar a variedade $W=\frac{M \times I}{\theta}$, para a qual temos

$$
\partial W=\partial\left(\frac{M \times I}{\theta}\right)=\frac{\partial M \times I}{\theta} \cup \frac{M \times \partial I}{\theta}=\frac{M \times\{0,1\}}{\theta} \cong M .
$$

Considere agora uma involução diferenciável $(T, M)$ sobre uma variedade diferenciável possivelmente desconexa. Fixe daqui em diante uma métrica Riemmaniana sobre $M^{n}$ com respeito a qual $T$ é uma isometria.

Denote $F=U F^{i}$, onde

$F$ é o conjunto dos pontos fixos de $T$.

$F^{i}$ é a união das componentes $i$-dimensionais do conjunto dos pontos fixos $(0 \leq i \leq n)$.

$\mathrm{O}$ conjunto $F^{i}$ é uma subvariedade $i$-dimensional mergulhada regularmente em $M^{n}$. 
Observe que $F^{n}$ consiste das componentes de $M^{n}$ que são ponto a ponto fixadas por $T$, não tendo portanto fibrado normal. Note também que uma vizinhança tubular $N$ de $F$ é uma união disjunta de vizinhanças tubulares em torno de cada componente de $F$.

Denote

$$
\begin{aligned}
& \nu_{i}=\nu\left(F^{i}, M\right) \rightarrow F^{i} \text { o fibrado normal a } F^{i} \text { em } M^{n}, i<n . \\
& q: S\left(\nu_{i}\right) \rightarrow F^{i} \text { o (n-i-1)-fibrado esfera diferenciável correspondente. }
\end{aligned}
$$

Para cada $i<n$ existe um fibrado involução $\left(T, S\left(\nu_{i}\right)\right)$ do fibrado esfera $S\left(\nu_{i}\right)$.

Denotemos

$$
(T, S(\nu))=\bigcup_{i<n}\left(T, S\left(\nu_{i}\right)\right)
$$

a união disjunta, onde chamamos

$S(\nu)$ o fibrado esfera normal ao conjunto dos pontos fixos.

Temos então

$$
[T, S(\nu)]_{2}=\sum_{i=0}^{n-1}\left[T, S\left(\nu_{i}\right)\right]_{2} \in \mathcal{N}_{n-1}\left(\mathbb{Z}_{2}\right) .
$$

Considerando agora $F^{n}=\varnothing, N$ uma vizinhança tubular de $F$ e denotando $B^{n}=M^{n}-N$, temos que $B^{n}$ é uma subvariedade com bordo $S(\nu)^{n-1}$ mergulhada regularmente em $M$, sobre a qual $T$ não tem pontos fixos. Assim, por definição, $[T, S(\nu)]_{2}$ borda, isto é,

$$
[T, S(\nu)]_{2}=\left[T, \partial B^{n}\right]_{2}=0 \quad \text { em } \quad \mathcal{N}_{n-1}\left(\mathbb{Z}_{2}\right)
$$

Finalmente chegamos ao Teorema de Conner-Floyd [4, Teorema 24.2].

Teorema 2.5.2. Seja $\left(T, M^{n}\right)$ uma involução diferenciável sobre uma variedade fechada. Denote por $q: \nu \rightarrow F$ o fibrado normal ao conjunto de pontos fixos $F$, por $q^{\prime}: \nu \oplus 1 \rightarrow F \quad$ a soma de Whitney de $\nu$ com o fibrado linha trivial, $e$ por $S(\nu \oplus 1)$ o fibrado esfera associado a $q^{\prime}$. Então

$$
\left[M^{n}\right]_{2}=[\operatorname{IRP}(\nu \oplus 1)]_{2}
$$




\subsection{Fibrado Linha Gerado por uma Subvarieda- de 1-Codimensional}

$\mathrm{Na}$ tentativa de obter condições para que uma $\mathbb{Z}_{2}$-variedade $M$ tenha classe de bordismo nula consideramos inicialmente o caso especial em que o conjunto dos pontos fixos é 1-codimensional. A técnica empregada aqui é baseada em Wasserman, [14], que utiliza o fibrado linha gerado por uma subvariedade 1codimensional $A$ de uma variedade $M$. A condição obtida para que $M$ borde depende de uma certa classe de Stiefel-Whitney associada a $A$.

Em [14, def. 24] Wasserman mostra o seguinte resultado:

Proposição 2.6.1. Se A é uma subvariedade invariante fechada de codimensão 1 de uma $G$-variedade $M$, então o fibrado normal de $A$ em $M, p: \nu=\nu(A, M) \rightarrow A$, pode ser estendido a um fibrado linha sobre $M$ inteiro, $\bar{p}: \bar{\nu} \rightarrow M$. Mais ainda, $\bar{\nu}$ tem uma secção equivariante $\bar{s}: M \rightarrow \bar{\nu}$ que zera exatamente sobre $A$ e que é transversà à secção nula de $\nu$.

Prova. Identifiquemos uma vizinhança tubular invariante $\mathrm{U}$ de $\mathrm{A} \operatorname{com} \nu$, observando que cada ponto $a \in A$ é associado a um vetor nulo $(a, 0)$ e que cada ponto $x \in U-A$ é associado a um vetor não-nulo.

Considerando o fibrado induzido, podemos estender $p: \nu \rightarrow A$ ao fibrado linha $p^{\prime} \nu \rightarrow \nu$, isto é,

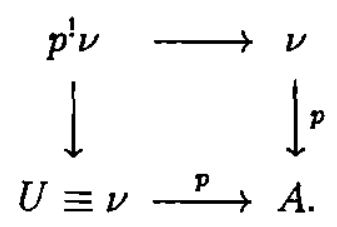

Considerando a secção canônica em $p^{!} \nu$, isto é, $s: \nu \rightarrow p^{!} \nu$, dada por $s(x)=$ $(x, x)$, esta zera exatamente sobre A. Daí, por 1.4.14, o fibrado linha $p^{\prime} \nu$ é trivial fora de A. Assim, basta estender $p^{\prime} \nu$ a um fibrado linha $\bar{\nu} \stackrel{\vec{p}}{\rightarrow} M$ colocando $\bar{\nu}_{\mid(M-U)}=(M-U) \times \mathbb{R}$. 
Definição 2.6.2. O G-fibrado $\bar{\nu} \rightarrow M$ obtido na proposição acima é chamado de $G$-fibrado linha gerado por $\boldsymbol{A}$.

Considerando a inclusão $i: A^{n-1} \longrightarrow M^{n}$ temos a aplicação induzida em homologia

$$
i_{*}: H_{n-1}\left(A ; \mathbb{Z}_{2}\right) \rightarrow H_{n-1}\left(M ; \mathbb{Z}_{2}\right)
$$

que leva $[A]$, a classe fundamental de $\mathrm{A}$, em $i_{*}[A]$.

Por outro lado, pelo Teorema de Dualidade de Poincaré Mod 2 [9, p.213], a aplicação

$$
H^{1}\left(M ; \mathbb{Z}_{2}\right) \stackrel{-[M]}{\longrightarrow} H_{n-1}\left(M ; \mathbb{Z}_{2}\right)
$$

dada por $x \rightarrow x \frown[M]$ é um isomorfismo.

Assim, conforme Wasserman [14, Obs.26] ou Milnor [11, Exercício 11C, pag. 135], temos

Lema 2.6.3. Sejam $i: A^{n-1} \rightarrow M^{n}$ um mergulho entre variedades compactas, $\nu=\nu(A, M)$ o fibrado linha normal a $A$ e $\bar{\nu}$ o fibrado linha gerado por $A$. Então $i_{*}[A]$ representa $w_{1}(\breve{\nu})$, isto é,

$$
i_{*}[A]=w_{1}(\bar{\nu}) \frown[M]
$$

Prova. Usando a nossa notação temos por Koschorke [7, Fato 9.7, pag.97] que para todo $c \in H^{n-1}\left(M ; \mathbb{Z}_{2}\right)$ vale a relação

$$
<i^{*}(c),[A]>=<c \smile w_{1}(\bar{\nu}),[M]>
$$

O primeiro termo da equação pode ser escrito como $\left\langle i^{*}(c),[A]\right\rangle=<c, i_{*}[A]>$. Para o segundo termo temos a relação [9, pag.190]

$$
<c \smile w_{1}(\bar{\nu}),[M]>=<c, w_{1}(\bar{\nu}) \frown[M]>, \forall c .
$$


Daí, obtemos

$$
<c, i_{*}[A]>=<c, w_{1}(\bar{\nu}) \frown[M]>, \forall c
$$

ou seja,

$$
i_{*}[A]=w_{1}(\bar{\nu}) \frown[M]
$$

Temos então

Proposição 2.6.4. Sejam $M$ uma $\mathbb{Z}_{2}$-variedade diferenciável conexa compacta, $F=\operatorname{Fix}\left(\mathbb{Z}_{2}, M\right)$ 1-codimensional conexo e $\bar{\nu}$ o fibrado linha gerado por $F$. Se $w_{1}(\bar{\nu})=0$ então $M-F$ possui duas componentes conexas.

Prova. Como $w_{1}(\bar{\nu})=0$, pelo Lema 2.6.3, o homomorfismo $i_{*}$ é nulo no nível 1 , e assim o seu transposto $i^{*}$ no nível $n-1$ também é nulo. Pelo Teorema de Dualidade de Lefschetz temos $H_{0}\left(M-F ; \mathbb{Z}_{2}\right) \simeq H^{n}\left(M, F ; \mathbb{Z}_{2}\right)$. Considerando a seqüência exata de cohomologia do par $(M, F)$, com coeficientes em $\mathbb{Z}_{2}$,

$$
\cdots \rightarrow H^{n-1}(M) \stackrel{\|^{*}=0}{\longrightarrow} H_{\mathbb{Z}_{2}^{n-1}}(F) \rightarrow H^{n}(M, F) \rightarrow \underset{\mathbb{Z}_{2}}{H^{n}(M)} \rightarrow \underset{\|}{H^{n}(F)} \rightarrow \cdots,
$$

obtemos $H^{n}\left(M, F ; \mathbb{Z}_{2}\right)=\mathbb{Z}_{2} \oplus \mathbb{Z}_{2}$. Daí $H_{0}\left(M-F ; \mathbb{Z}_{2}\right) \simeq \mathbb{Z}_{2} \oplus \mathbb{Z}_{2}$, isto é, $M-F$ tem duas componentes conexas.

Denotemos então as duas componentes conexas de $M-F$ por $W_{1} e W_{2}$. Como $\mathbb{Z}_{2}$ atua livremente em $M-F=W_{1} \cup W_{2}$, temos uma involução livre

$$
T: W_{1} \dot{\cup} W_{2} \rightarrow W_{1} \dot{\cup} W_{2}
$$

Como a ação de $\mathbb{Z}_{2}$ no fibrado normal manda $\mathrm{x}$ em $-\mathrm{x}$, temos que $x \in W_{1}$ é levado em $T(x) \in W_{2}$ e vice-versa. Daí, por conexidade, $W_{1} e W_{2}$ são homeomorfas.

Denotemos $W=\bar{W}_{1} \simeq \bar{W}_{2}$, onde $\bar{W}_{i}$ denota o fecho de $W_{i}$. Temos então:

$$
\partial W=F \quad \text { e } \quad M=W \cup_{\partial} W=2 W,
$$


onde $2 W$ denota o dobro de $W$.

A variedade dobro de $W$ tem uma propriedade muito importante

Lema 2.6.5. Se $W^{n}$ é uma variedade com bordo, então $2 W$ é bordo de uma variedade $(n+1)$-dimensional $V$.

Prova. Consideremos a variedade com cantos $V=W \times I$, onde $I=[-1,+1]$. Temos

$$
\partial V=\partial(W \times I)=\partial W \times I \bigcup_{I d_{\theta W \times \theta I}} W \times \partial I=\partial W \times I \bigcup_{I d_{\partial W \times \theta I}} W \times S^{0} \equiv 2 W,
$$

onde a segunda igualdade é o bordo do produto cartesiano de duas variedades com bordo [3, 13.13].

Uma visão geométrica deste lema é esboçada abaixo.

Observe inicialmente que colar duas $W^{n}$ pelo bordo é equivalente a colar duas $W$ em $\partial W \times I$.

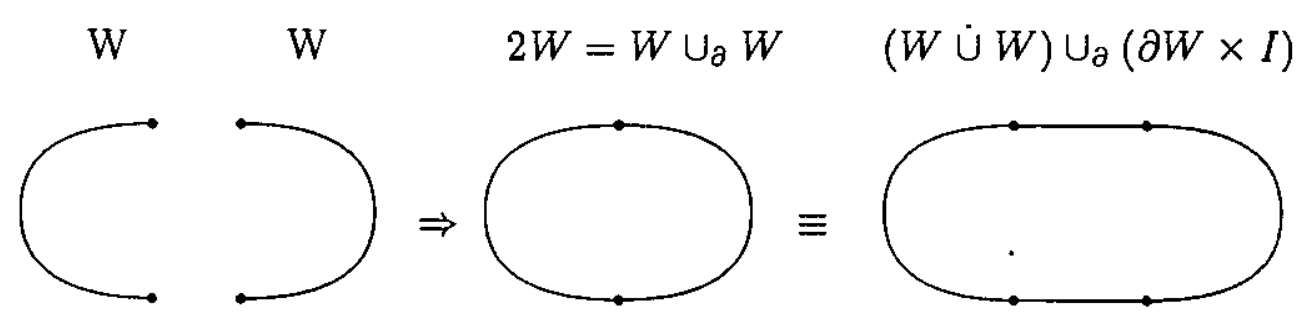

(Para a diferenciabilidade a colagem deve ser feita sobrepondo duas vizinhanças tubulares de $\partial W$.)

Considerando dois dobros de $W$, podemos repetir o processo acima e "preencher" os espaços delimitados obtendo uma $(n+1)$-variedade $V$. 


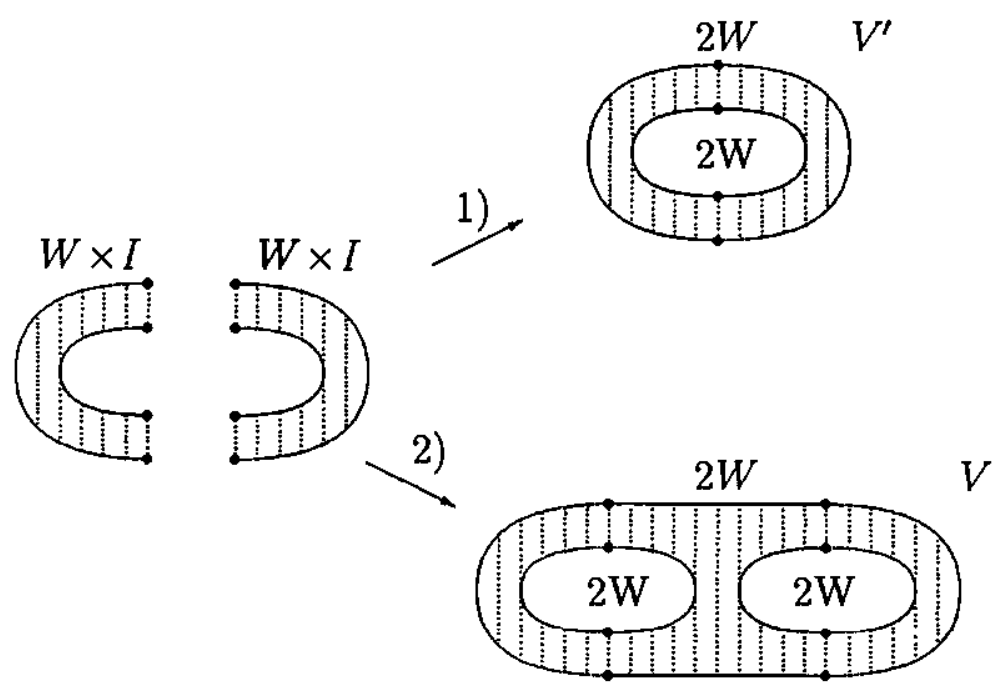

Aqui $V^{\prime}=(W \times I) \cup_{\partial W \times I}(W \times I)$.

Temos então dois casos:

1) $\partial V^{\prime}=2 W \dot{\cup} 2 W \quad \Longrightarrow \quad\left[\partial V^{\prime}\right]_{2}=[2 W \dot{\cup} 2 W]_{2}=[2 W]_{2}+[2 W]_{2}=0 \bmod 2$;

2) $\partial V=2 W \dot{\cup} 2 W \dot{\cup} 2 W \quad \Longrightarrow \quad[\partial V]_{2}=[2 W \dot{\cup} 2 W]_{2}+[2 W]_{2} \stackrel{1)}{=}[2 W]_{2}$.

Obtivemos assim a variedade $V$ com bordo $2 W$.

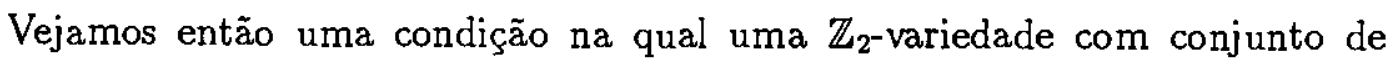
pontos fixos 1-codimensional borda.

Teorema 2.6.6. Sejam $M$ uma $\mathbb{Z}_{2}$-variedade diferenciável conexa compacta, $F=\operatorname{Fix}\left(\mathbb{Z}_{2}, M\right)$ conexo 1-codimensional e $\bar{\nu}$ o fibrado linha gerado por $F$. Se $w_{1}(\bar{\nu})=0$ então existe uma variedade $V$ com $\quad \partial V=M$.

Prova. Pela Proposição 2.6.4 $M-F$ tem duas componentes. Considerando $M-F=W_{1}$ ப் $W_{2}$ vimos acima que a involução livre atuando em $W_{1} \dot{\cup} W_{2}$ faz $\bar{W}_{1} \equiv \bar{W}_{2}=W, \partial W=F$ e $M=2 W$. Daí, pelo Lema 2.6.5, existe uma variedade $\mathrm{V}$ com $\partial V=2 W$, isto é, $\partial V=M$.

Segue imediatamente do Teorema de Pontrjagin, 2.3.2, que nas condições acima $W_{I}(M)=0$ para toda partição $I$ de $n$. 
O mais importante é que o teorema absoluto acima pode ser estendido ao caso em que a variedade bordante $V$ também é uma $\mathbb{Z}_{2}$-variedade tendo conjunto de pontos fixos 1-codimensional.

Teorema 2.6.7. Sejam $M$ uma $\mathbb{Z}_{2}$-variedade diferenciável conexa compacta, $F=\operatorname{Fix}\left(\mathbb{Z}_{2}, M\right)$ conexo 1-codimensional e $\bar{\nu}$ o fibrado linha gerado por $F$. Se $w_{1}(\bar{\nu})=0$ então existe uma $\mathbb{Z}_{2}$-variedade tendo conjunto de pontos fixos 1 codimensional $V$ com $\partial V=M$.

Prova. Vimos acima que $V=W \times I$, onde $W=\bar{W}_{1} \equiv \bar{W}_{2}$ e $M-F=W_{1}$ نे $W_{2}$. Para dar uma estrutura de $\mathbb{Z}_{2}$-variedade a $V$ basta considerar uma ação $\phi$ em $V$ dada por

$$
\phi(w, t)=(w,-t), \quad \text { onde } \quad(w, t) \in W \times I .
$$

Daí, $V$ é uma $\mathbb{Z}_{2}$-variedade tendo conjunto de pontos fixos 1-codimensional pois

$$
V\left(\mathbb{Z}_{2}\right)=\operatorname{Fix}\left(\mathbb{Z}_{2}, V\right)=\{(w, 0) \in W \times I\} \equiv W
$$

No capítulo seguinte, usando a técnica do blow-up, verificamos que a condição $w_{1}(\bar{\nu})=0$ do teorema acima pode ser eliminada. 


\section{Capítulo 3}

\section{Blow-up e Ações Não-Singulares}

Inicialmente, baseados em Wasserman [14] e [15], definimos blow-up e ações nãosingulares. Em seguida determinamos relações entre a classe de bordismo de uma variedade $M$ e seu blow-up $B(A, M)$. Concluímos analisando bordismo no caso de açōes não-singulares.

\subsection{Blow-up}

Consideraremos aqui $G$ um grupo de Lie compacto. Se $\pi: E \rightarrow X$ é um $G$-fibrado vetorial, com uma métrica Riemanniana invariante, denotamos:

$D(E) \quad$ o fibrado disco de $E$;

$S(E) \quad$ o fibrado esfera unitária de $E$;

$\mathbb{R} P(E)=\frac{S(E)}{\mathbf{Z}_{2}} \quad$ o fibrado projetivo real de $E ;$

$L(E)=\frac{S(E) \times R}{\mathbb{Z}_{2}}$ o fibrado linha associado ao recobrimento $S(E) \rightarrow \mathbb{R} P(E)$.

$\mathbb{R P}(E)$ é obtido de $S(E)$ identificando $v \in S(E)$ com $-v$.

Se $A$ é uma subvariedade de uma $G$-variedade $M$ denotamos $\nu=\nu(A, M)$ o fibrado normal de $A$ em $M$.

Quando $A$ é fechada, invariante, $\partial A \subset \partial M$ e $A$ é transversa a $\partial M$ identificaremos $\nu$ com $V_{A}$, uma vizinhança tubular equivariante de $A$ em $M$. 
Considerando $A^{m} \longrightarrow M^{n}$ e $n-m=k$ podemos obter a partir do fibrado $\nu$ o fibrado $\operatorname{IRP}(\nu \oplus 1)$, isto é,

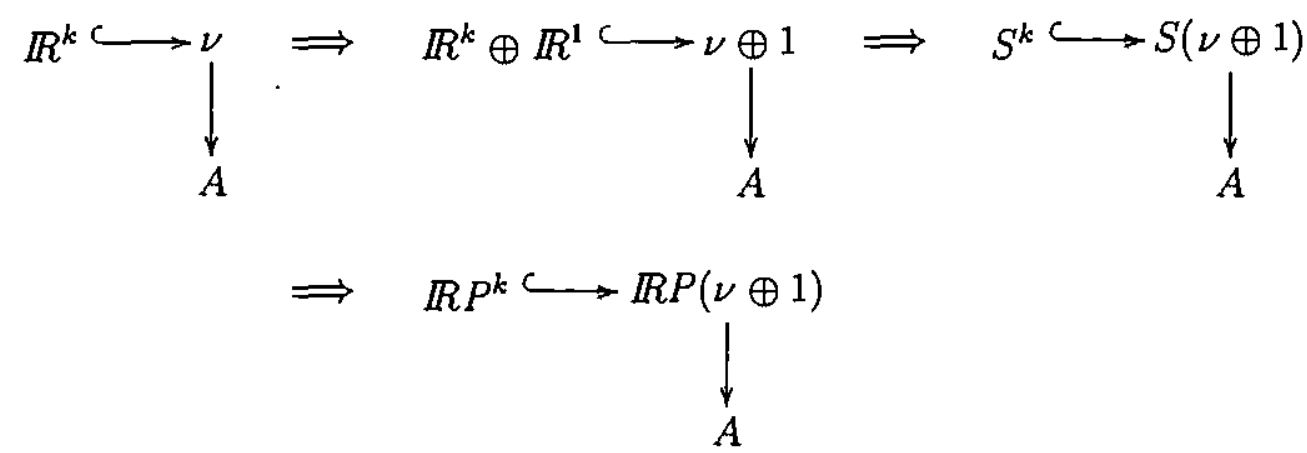

Considerando a ação sobre $\nu$ como sendo a ação obtida pela identificação de $\nu$ com a vizinhança tubular equivariante de $A$, e considerando sobre o espaço total do fibrado linha trivial 1 a ação trivial, obtemos uma ação sobre $\nu \oplus 1$ que induz uma ação sobre o espaço $\operatorname{IRP}(\nu \oplus 1)$, tornando-o uma $G$-variedade.

Agora podemos definir o blow-up.

Definição 3.1.1. Se A é uma subvariedade invariante fechada da G-variedade $M$ com $\partial A \subset \partial M$ e A transversa a $\partial M$, definimos o blow-up equivariante de $A$ em $M$ por

$$
B(A, M)=\left\{\begin{array}{lll}
M \#_{A} I R P(\nu \oplus 1), & \text { se } & \operatorname{dim} A<\operatorname{dim} M \\
M & \text { se } & \operatorname{dim} A=\operatorname{dim} M
\end{array}\right.
$$

onde $\#_{A}$ é a soma conexa ao longo de $A$.

O blow-up $B(A, M)$ é uma $G$-variedade diferenciável pois a soma conexa ao longo de $A$ na definição acima é obtida pela colagem, ao longo dos bordos, de $\left(M-V_{A}\right)$ com $\left(\operatorname{IRP}(\nu \oplus 1)-V_{A}\right)$ usando o Teorema do Colarinho Equivariante. ( $[2$, Teorema V.1.5] ou [4, Teorema 21.2].)

A construção blow-up pode ser esboçada geometricamente como descrito abaixo. 
Denotando

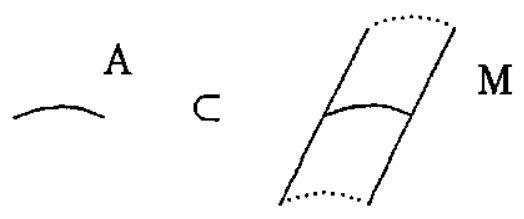

temos

$\nu \quad \nu \oplus 1 \quad S(\nu \oplus 1) \quad \operatorname{IRP}(\nu \oplus 1) \quad M \#_{A} \operatorname{IRP}(\nu \oplus 1)=B(A, M)$
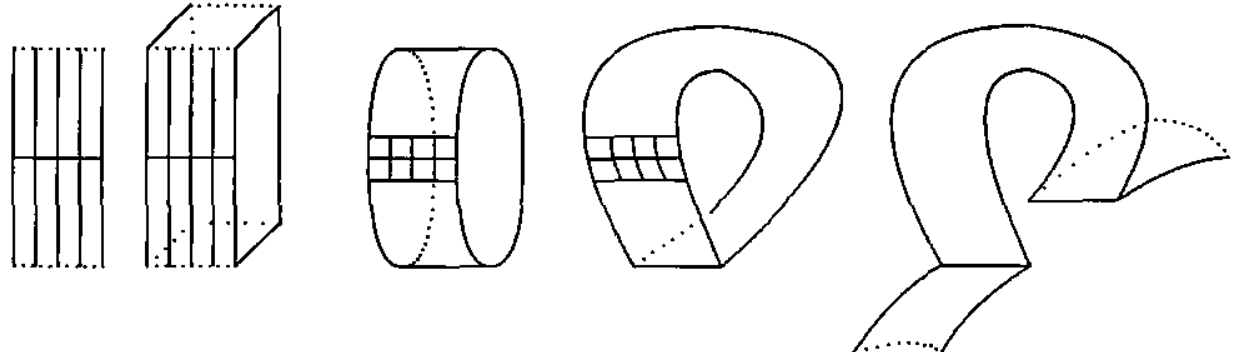

Podemos também obter $L(\nu)$ a partir de $\nu$ como segue:

Primeiro obtemos os fibrados $S(\nu)$ e $I R P(\nu)$ :

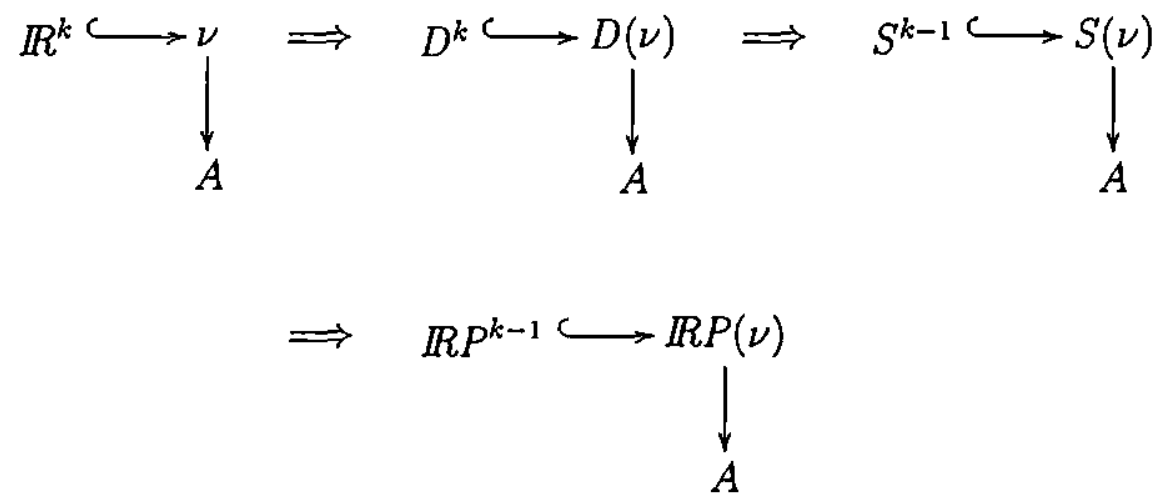

Daí, obtemos $L(\nu)$ como o fibrado linha associado ao $\mathbb{Z}_{2}$-fibrado principal $S(\nu) \rightarrow \operatorname{IRP}(\nu)$ 


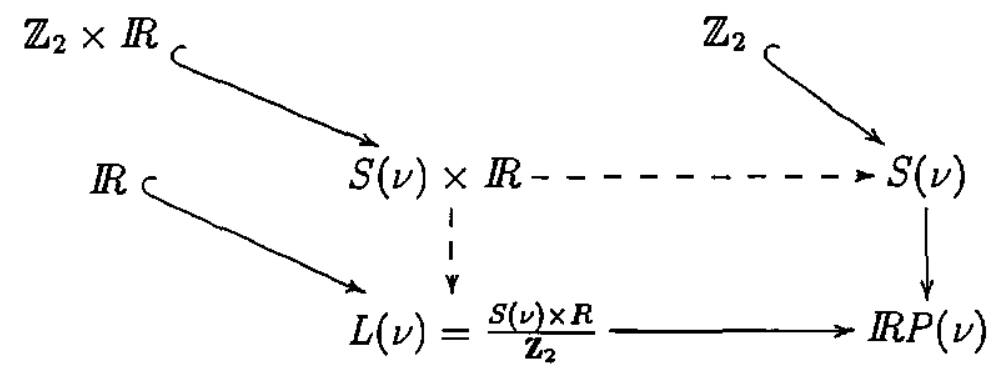

Ou seja,

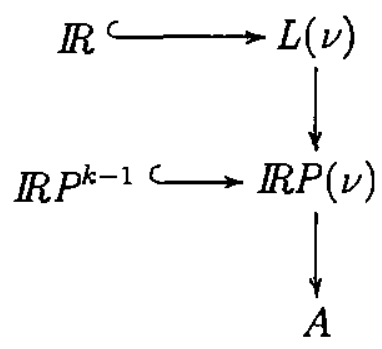

Agora, restringindo a fibra $I R$ à $\mathbb{Z}_{2}=\{+1,-1\} \subset \mathbb{R}$, isto é,

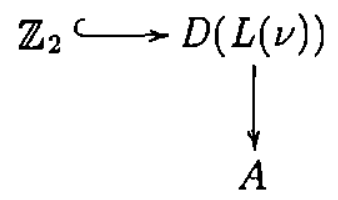

podemos dar uma definição alternativa do blow-up:

$$
B(A, M)=[M-\stackrel{\circ}{D}(\nu)] \cup_{f} D(L(\nu)),
$$

onde a aplicação colagem $f: S(L(\nu)) \rightarrow S(\nu)$ é a aplicação identidade.

Geometricamente, considerando $A$ e $M$ como antes, temos:

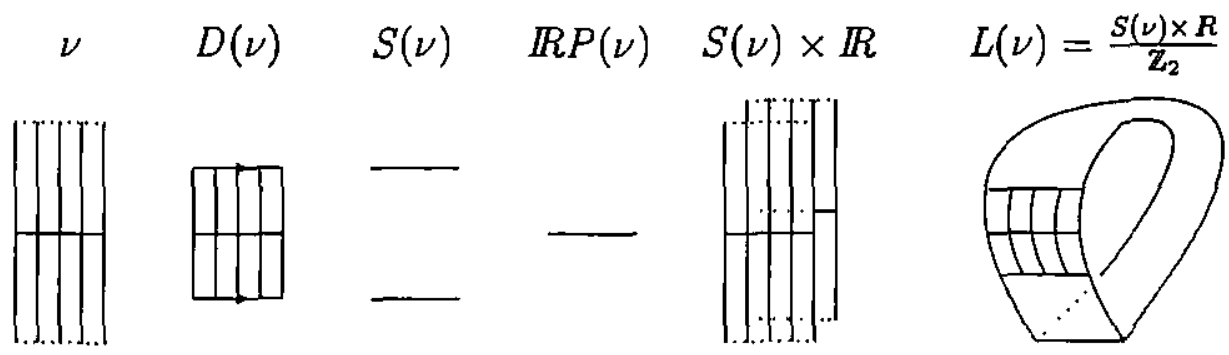




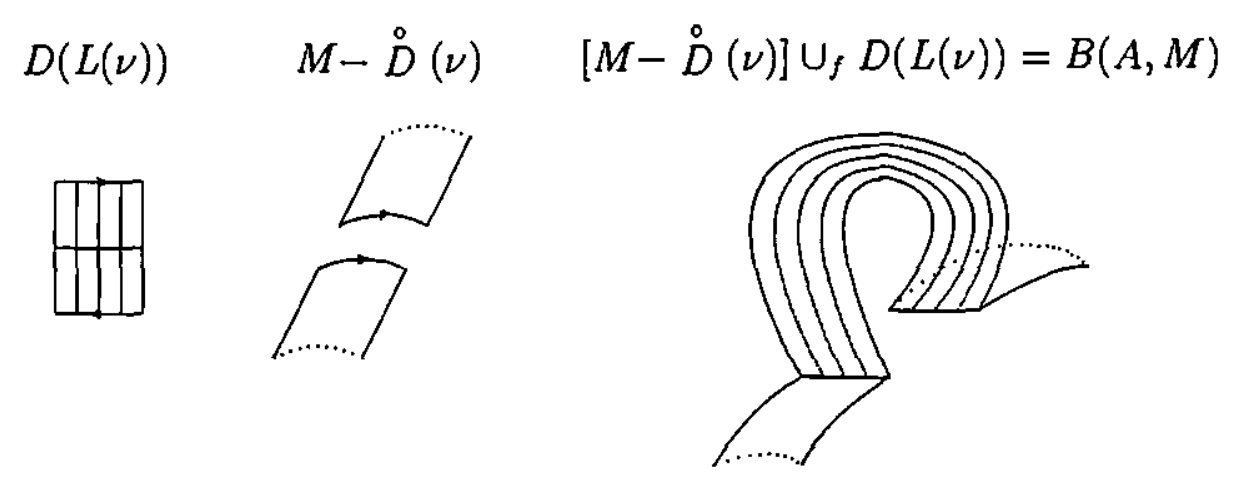

Informalmente, $B(A, M)$ é obtido de $M$ deletando os pontos de $A$ e adicionando retas normais a $A$ em $M(\operatorname{Se} \operatorname{dim} A<\operatorname{dim} M)$.

Exemplo 3.1.2. Seja $M=S^{1}$ e $A=\{*\}$.

Temos

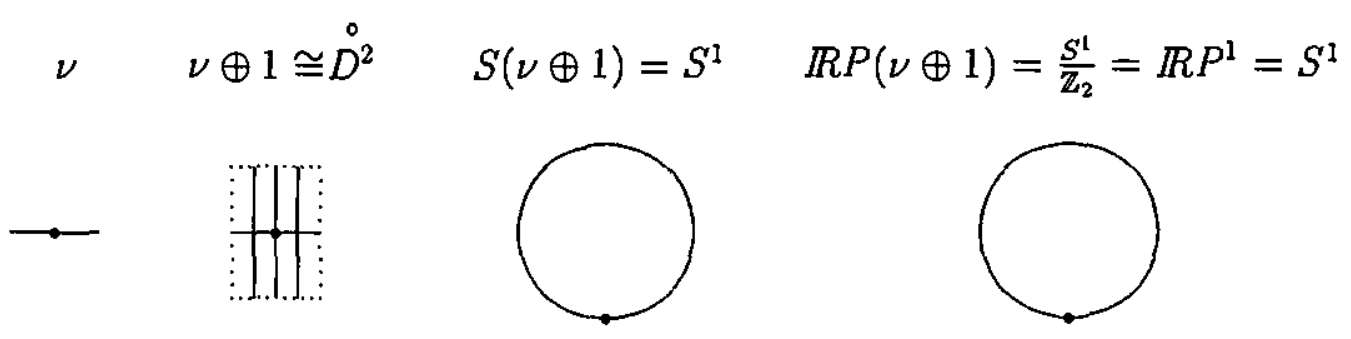

Assim

$$
B(A, M)=M \underset{A}{\#} \mathbb{R} P(\nu \oplus 1)=S_{\{*\}}^{1} S^{1}=S^{1} .
$$

Observamos que a soma conexa ao longo de $A=\{*\}$ é a soma conexa usual.

Exemplo 3.1.3. Seja $M=S^{2}$ e $A=\{*\}$.

Temos 


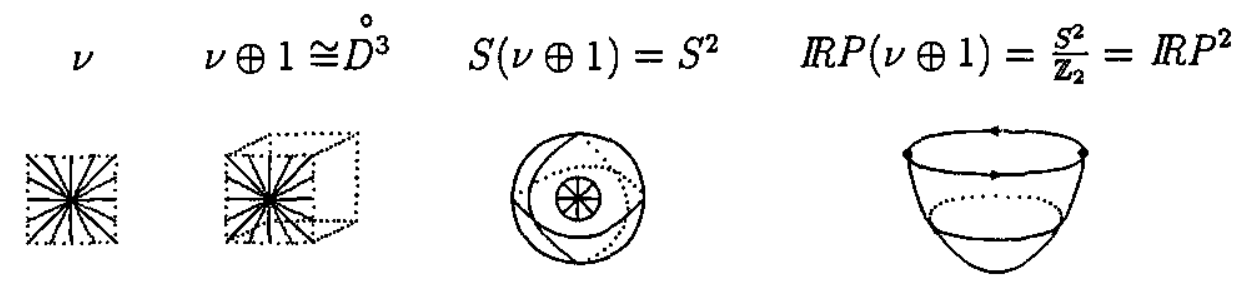

Assim

$$
B(A, M)=S^{2} \underset{\{*\}}{\#} \mathbb{I R} P^{2}=\mathbb{R} P^{2}
$$

Mais geralmente, para $M=S^{n}$ e $A=\{*\}$ temos $\mathbb{R} P(\nu \oplus 1)=\mathbb{R} P^{n}$, e assim

$$
B(A, M)=S_{\{*\}}^{\#} \mathbb{R} P^{n}=\mathbb{R} P^{n}
$$

Exemplo 3.1.4. Seja $M=S^{2}$ e $A=S^{0}$.

Considerando $A_{1}=\{*\} \quad$ e $\nu_{1}=\nu\left(A_{1}, M\right)$, vimos no Exemplo 3.1 .3 que

$$
\mathbb{R} P\left(\nu_{1} \oplus 1\right)=\mathbb{R} P^{2}
$$

Assim

$$
\begin{aligned}
B(A, M) & =S^{2} \#_{A} \mathbb{I R P}(\nu \oplus 1)=S^{2} \#_{A}\left(\mathbb{I R} P^{2} \dot{\cup} \mathbb{I R} P^{2}\right)=\mathbb{R} P^{2} \# S^{2} \# \mathbb{I} P^{2} \\
& =\mathbb{R} P^{2} \# \mathbb{R} P^{2}=K b,
\end{aligned}
$$

onde $K b$ denota a garrafa de Klein.

Exemplo 3.1.5. Seja $M=S^{2}$ e $A=S^{1}$.

Temos

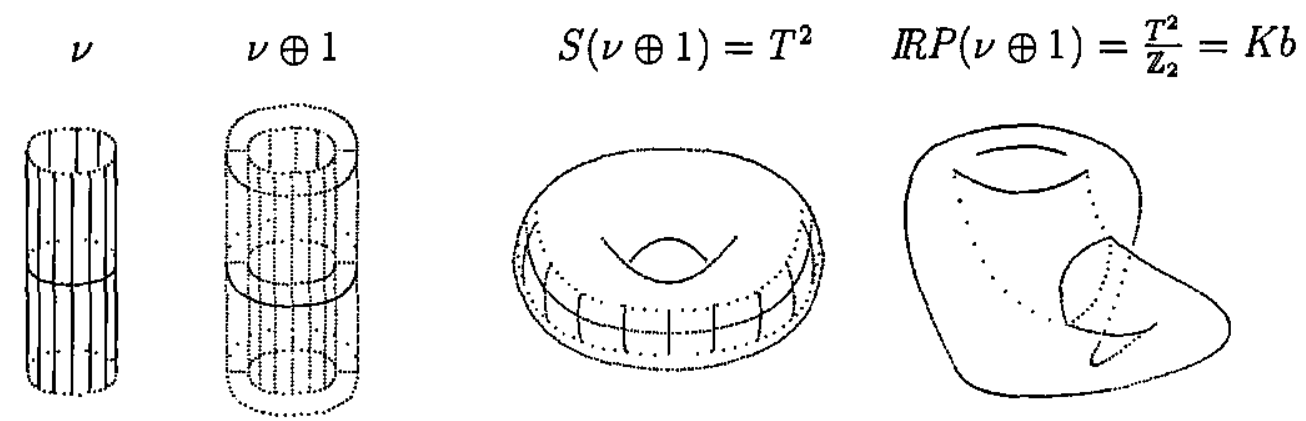


onde $T^{2}$ denota o toro. Assim

$$
B(A, M)=S_{S^{1}}^{2} \# b=\left[S^{2}-V_{S^{1}}\right] \cup_{\partial}\left[K b-V_{S^{1}}^{\prime}\right]=S^{2},
$$

onde $V_{S^{1}}$ e $V_{S^{1}}^{\prime}$ são vizinhanças de $S^{1}$ em $S^{2}$ e em $K b$.

Observamos aqui que a soma conexa ao longo de $S^{1}$ de uma variedade orientável com uma não-orientável resultou em uma orientável, fato que não ocorre na soma conexa usual.

Apesar de estarmos interessados apenas em variedades fechadas, vejamos um exemplo onde a variedade $M$ tem bordo.

Exemplo 3.1.6. Seja $M=D^{2}$ o disco e $A=\{*\}$ um ponto de seu interior.

Como $\operatorname{RP} P(\nu \oplus 1)=\mathbb{R} P^{2}$ temos

$$
B(A, M)=D^{2} \# \mathbb{Z} \underset{\{*\}}{I R P^{2}}=\mathbb{I R P} P^{2}-\stackrel{\circ}{D^{2}}=F M,
$$

onde FM denota a faixa de Möbius.

O blow-up de $A$ em $M$ é apenas uma parte de uma construção mais geral que é o blow-up do conjunto de todos os pontos fixos de uma $G$-variedade $M$, como descrevemos abaixo.

Seja $M$ uma $G$-variedade e denotemos

$$
\begin{aligned}
& F=\operatorname{Fix}(G, M)=\dot{U} F_{i}=F_{1} \dot{U} \cdots \dot{U} F_{r}, \\
& \nu=\nu(F, M)=\sum_{i=1}^{r} \nu\left(F_{i}, M\right)=\sum_{i=1}^{r} \nu_{i} .
\end{aligned}
$$

O blow-up do conjunto dos pontos fixos $F$ de $M$ é dado por

$$
B(F, M)=B\left(F_{r}, B\left(F_{r-1}, \ldots B\left(F_{2}, B\left(F_{1}, M\right)\right) \ldots\right)\right)
$$

ou seja,

$$
B(F, M)=\left(\ldots\left(\left(M \underset{F_{1}}{\#} \mathbb{I} P\left(\nu_{1} \oplus 1\right)\right) \underset{F_{2}}{\#} \mathbb{R} P\left(\nu_{2} \oplus 1\right)\right) \underset{F_{3}}{\#} \ldots\right) \underset{F_{r}}{\#} \mathbb{R} P\left(\nu_{r} \oplus 1\right) .
$$


Em particular, quando $F=S^{0}=\left\{P_{1}, P_{2}\right\} \subset M$ temos a decomposição

$$
B\left(S^{0}, M\right)=B\left(P_{2}, B\left(P_{1}, M\right)\right)
$$

Observamos que apesar de podermos calcular $B(A, M)$, para uma dada subvariedade invariante $A$ de $M$, isto não implica que $M$ tenha $A$ como o seu conjunto de pontos fixos.

Completemos agora os casos possíveis de blow-up em dimensão 2.

Recordemos que, pelo Teorema de Classificação de Superfícies Compactas, ver por exemplo [8], toda variedade compacta conexa 2-dimensional, $M^{2}$, é homeomorfa a uma esfera $S^{2}$, ou a uma soma conexa de toros $T^{2}$, ou a uma soma conexa de planos projetivos $\mathbb{R} P^{2}$.

Mais ainda, em termos de orientação temos:

$M^{2}$ orientada $\quad \Rightarrow M^{2}=S^{2}$ ou $M^{2}=T^{2} \# \ldots \# T^{2}=\left(T^{2}\right)^{r}, r \geq 1$;

$M^{2}$ não orientada $\Rightarrow M^{2}=\mathbb{R} P^{2} \# \ldots \# \mathbb{R} P^{2}=\left(\mathbb{R} P^{2}\right)^{r}, r \geq 1$.

Em termos de bordismo temos:

$\left[M^{2}\right]_{2}=0 \Rightarrow M^{2}=S^{2}$, ou $M^{2}=\left(T^{2}\right)^{r}$ ou $M^{2}=(K b)^{r}=\left(\mathbb{R} P^{2}\right)^{2 r}, r \geq 1 ;$ $\left[M^{2}\right]_{2} \neq 0 \Rightarrow M^{2}=\mathbb{R} P^{2} \# \ldots \# \mathbb{R} P^{2}=\left(\mathbb{R} P^{2}\right)^{2 r-1}, r \geq 1$.

Prosseguindo no cálculo dos blow-ups, como em exemplos anteriores, obtemos todos os blow-ups parciais possíveis em dimensão 2 a partir dos três casos abaixo:

1) $B\left(*, M^{2}\right)=M \#_{\{*\}} I R P^{2}=M \# \mathbb{R} P^{2}$.

2) $B\left(S^{0}, M^{2}\right)=M \#\left\{\mathbb{R} P^{2} \dot{\cup} \mathbb{R} P^{2}\right\}=M \# K b$ :

2.1) $[M]_{2}=0 \Rightarrow B\left(S^{0}, M^{2}\right)=M \# K b=M \#\left(\mathbb{R} P^{2}\right)^{2}$;

2.2) $[M]_{2} \neq 0 \Rightarrow B\left(S^{0}, M^{2}\right)=M \# K b=\left(\mathbb{R} P^{2}\right)^{2 r-1} \#\left(\mathbb{R} P^{2}\right)^{2}=\left(\mathbb{R} P^{2}\right)^{2 r+1}$. 
3) $B\left(S^{1}, M^{2}\right)=M \#_{S^{1}} \mathbb{R P}(\nu \oplus 1)=M \#_{S^{1}} K b=\left(M-V_{S^{1}}\right) \cup_{\partial}\left(K b-V_{S^{1}}\right)$ :

3.1) $S^{1} \stackrel{i}{\hookrightarrow} M^{2}$ mergulho homotopicamente nulo: $\quad B\left(S^{1}, M^{2}\right)=M^{2}$.

3.2) $S^{1} \stackrel{i}{\hookrightarrow} M^{2}$ mergulho homotopicamente não-nulo:

3.2.1) $S^{1} \stackrel{\leftrightarrow}{\leftrightarrow} F M \subset M^{2}$ (mergulho).

$$
\begin{aligned}
& B\left(S^{1}, M^{2}\right)=\left(M-V_{S^{1}}\right) \cup_{\partial F M} F M=M^{2} . \text { Assim: } \\
& B\left(S^{1},\left(\mathbb{R} P^{2}\right)^{r}\right)=\left(\mathbb{R} P^{2}\right)^{r} .
\end{aligned}
$$

3.2.2) $S^{1} \stackrel{i}{\hookrightarrow} S^{1} \times I \subset M^{2}$ (mergulho direto).

$B\left(S^{1}, M^{2}\right)=\left(M-V_{S^{1}}\right) \cup_{\partial \text { Cil }}$ Cil (desorientado). Assim:

a) $B\left(S^{1},\left(T^{2}\right)^{r}\right)=(K b)^{r}$;

b) $B\left(S^{1},(K b)^{r}\right)=\left(T^{2}\right)^{r}$ ou $B\left(S^{1},(K b)^{r}\right)=(K b)^{r}$ para $r>1$;

c) $\left.B\left(S^{1},\left(\mathbb{R} P^{2}\right)^{2 r-1}\right)\right)=\left(\mathbb{R} P^{2}\right)^{2 r-1}$ para $r>1$.

Aqui $V_{S^{1}}$ denota uma vizinhança de $S^{1}$ em $M^{2}$ ou em $\operatorname{IRP}(\nu \oplus 1)$ e Cil $=S^{1} \times I$.

Podemos interpretar geometricamente os resultados acima como:

1) O blow-up de um ponto em $M^{2}$ cola um espaço projetivo na variedade $M$, tornando-a uma variedade não-orientável, caso ainda não o seja. Especificamente temos:

$$
\begin{aligned}
& B\left(*, S^{2}\right)=S^{2} \# \mathbb{R} P^{2}=\mathbb{R} P^{2} \\
& B\left(*,\left(T^{2}\right)^{r}\right)=\left(T^{2}\right)^{r} \# \mathbb{R} P^{2}=\left(\mathbb{R} P^{2}\right)^{2 r+1} \\
& B\left(*,\left(\mathbb{R} P^{2}\right)^{r}\right)=\left(\mathbb{R} P^{2}\right)^{r} \# \mathbb{R} P^{2}=\left(\mathbb{R} P^{2}\right)^{r+1}
\end{aligned}
$$

2) O blow-up de uma esfera $S^{0}$ em $M^{2}$ cola uma garrafa de Klein na variedade $M$, tornando-a uma variedade não-orientável, caso ainda não o seja. Especificamente temos:

$$
\begin{aligned}
& B\left(S^{0}, S^{2}\right)=S^{2} \# K b=K b=\left(\mathbb{R} P^{2}\right)^{2} \\
& B\left(S^{0},\left(T^{2}\right)^{r}\right)=\left(T^{2}\right)^{r} \# K b=(K b)^{r+1}=\left(\mathbb{R} P^{2} \# \mathbb{R} P^{2}\right)^{r+1}=\left(\mathbb{R} P^{2}\right)^{2 r+2} \\
& B\left(S^{0},\left(\mathbb{R} P^{2}\right)^{r}\right)=\left(\mathbb{R} P^{2}\right)^{r} \# K b=\left(\mathbb{R} P^{2}\right)^{r+2} .
\end{aligned}
$$


3) O blow-up de $S^{1}$ em $M^{2}$ apenas modifica a variedade $M^{2}$ quando $S^{1}$ é um mergulho direto, $S^{1} \stackrel{i}{\hookrightarrow} S^{1} \times I \subset M^{2}$, homotopicamente não-nulo. Nestes casos o blow-up de $S^{1}$ em $M^{2}$ transforma uma soma conexa de $r$ toros em uma soma conexa de $r$ garrafas de Klein e vice-versa. Para $r>1$ podemos ter também $B\left(S^{1},(K b)^{r}\right)=(K b)^{r}$.

Esquematicamente, denotando $B_{0}=B(*, \quad)$ e $B_{1}=B\left(S^{1}, \quad\right)$, temos

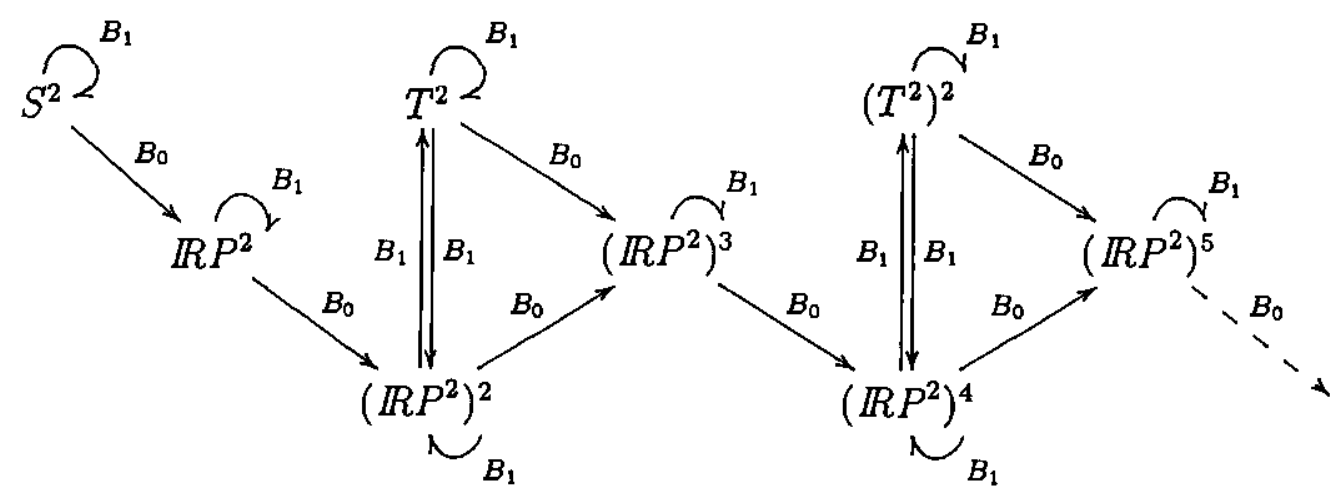

Em termos da característica de Euler correspondente a cada variedade temos

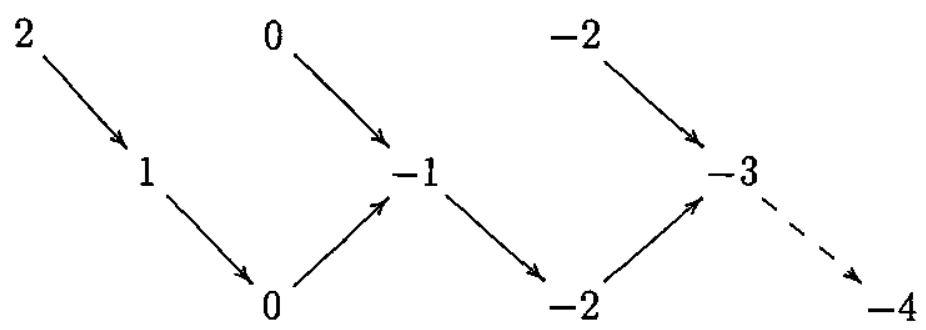




\subsection{Ações Não-Singulares}

Consideramos aqui $G$ um grupo de Lie compacto atuando sobre um espaço $X$ e $H$ um subgrupo fechado de $G$.

Denotamos por $X_{H}$ o conjunto dos pontos de $X$ com grupo de isotropia $\boldsymbol{H}$, isto é,

$$
X_{H}=\left\{x \in X \mid G_{x}=H\right\}
$$

Denotamos também

$$
X_{(H)}=G X_{H} .
$$

Observamos que

$$
X_{(H)} \equiv X(H)=\left\{x \in X \mid\left(G_{x}\right)=(H)\right\},
$$

o conjunto dos pontos de $\boldsymbol{X}$ com tipo de isotropia $\boldsymbol{H}$, definido na seção 1.2 , pois:

$$
\begin{aligned}
y \in X_{(H)} & \Leftrightarrow y=g x \quad e \quad G_{x}=H \Leftrightarrow G_{y}=G_{g x}=g G_{x} g^{-1} \quad \text { e } G_{x}=H \\
& \Leftrightarrow G_{y}=g H g^{-1} \Leftrightarrow\left(G_{y}\right)=(H) \Leftrightarrow y \in X(H) .
\end{aligned}
$$

Consideremos agora um tipo especial de ação muito importante.

Definição 3.2.1. Uma ação de $G$ em uma variedade $M$ com grupo de isotropia principal igual a $\{e\}$ é dita ação não-singular (e $M$ é dita $G$-variedade nãosingular) se $\forall x \in M$ temos:

i) $G_{x}$ é um $\mathbb{Z}_{2}$-espaço vetorial;

ii) $\operatorname{dim}_{\mathbb{Z}_{2}} G_{x}=\operatorname{codim} M\left(G_{x}\right)$.

Em particular, toda ação livre é não-singular. 
O exemplo seguinte de variedade não-singular ilustra bem os conceitos e propriedades das seções 1.2 e 1.3 do capítulo 1 .

Exemplo 3.2.2. Seja $M=S^{2}$ e $G=\mathbb{Z}_{2} \times \mathbb{Z}_{2}=\langle a, b\rangle$, onde a e $b$ são geradores de $\mathbb{Z}_{2} \times\{0\}$ e $\{0\} \times \mathbb{Z}_{2}$. Considere uma $G$-ação em $M$ dada por

$$
\begin{aligned}
& (a,(x, y, z)) \rightarrow a(x, y, z)=(-x, y, z) ; \\
& (b,(x, y, z)) \rightarrow b(x, y, z)=(x,-y, z) .
\end{aligned}
$$

Como $G$ é abeliano denotamos $\left(G_{x}\right)=G_{x}$. Os tipos de isotropia que ocorrem em $M$ são os $\mathbb{Z}_{2}$-espaços vetoriais:

$$
H_{1}=\mathbb{Z}_{2} \times \mathbb{Z}_{2}, \quad H_{2}=\mathbb{Z}_{2} \times\{0\}, \quad H_{3}=\{0\} \times \mathbb{Z}_{2}, \quad \text { e } H_{4}=\{0\} \times\{0\}=e .
$$

Mais ainda,

$$
\begin{aligned}
& M\left(H_{1}\right)=\{(0,0,1),(0,0,-1)\} \equiv\{N, S\}, \text { pólos, } \\
& M\left(H_{2}\right)=\left\{(0, y, z) \in S^{2} ; y^{2}+z^{2}=1 ; z \neq \pm 1\right\} \equiv S^{1}-\{N, S\}, \\
& M\left(H_{3}\right)=\left\{(x, 0, z) \in S^{2} ; x^{2}+z^{2}=1 ; z \neq \pm 1\right\} \equiv S^{1}-\{N, S\}, \\
& M\left(H_{4}\right)=S^{2}-M\left(H_{1}\right)-M\left(H_{2}\right)-M\left(H_{3}\right),
\end{aligned}
$$

e daí temos

$$
\operatorname{dim}_{\mathbb{Z}_{2}} H_{i}=\operatorname{codim} M\left(H_{i}\right), \quad \text { para } i=1,2,3,4,
$$

mostrando que $S^{2}$ com esta ação é uma $G$-variedade não-singular.

Em [14] Wasserman mostra que:

1. Ações com grupo de isotropia principal diferente de $\{e\}$ podem ser reduzidas a ações com grupo de isotropia igual a $\{e\}$;

2. Se o grupo de isotropia principal da ação é igual a $\{e\}$ então, através de um número finito de blow-ups equivariantes, a variedade $M$ pode ser reduzida a uma variedade $M^{\prime}$ que tem apenas finitos 2-grupos como grupos de isotropia; 
3. Quando o grupo $G$ é abeliano a ação pode ser simplificada a uma ação não-singular;

4. Se $M$ é uma $G$-variedade não-singular então o espaço quociente $\frac{M}{G}$ tem uma estrutura diferenciável. (Neste caso, geralmente $\frac{M}{G}$ é uma variedade com cantos.) 


\subsection{Classes de Bordismo de $M$ e $B(A, M)$}

Quando fazemos uma cirurgia em uma variedade fechada, a variedade resultante pertence à mesma classe de bordismo da variedade inicial [10, Teorema 1, pag.40] Em geral, isto não acontece quando fazemos um blow-up. Vimos no Exemplo 3.1.3 que $M=S^{2}$, com $A=\{*\}$, transformou-se após o blow-up no espaço projetivo $\mathbb{R} P^{2}$. Neste caso $S^{2}$ e $\mathbb{R} P^{2}$ não estão na mesma classe de bordismo. Vamos então determinar relações entre as classes de bordismo de $M$ e $B(A, M)$.

Como $B(A, M)=M \#_{A} \mathbb{R P}(\nu \oplus 1)$ temos

$$
[B(A, M)]_{2}=[M \underset{A}{\#} \mathbb{R} P(\nu \oplus 1)]_{2} .
$$

Em particular, quando $A=\{*\}$ temos a soma conexa usual. Neste caso vale

$$
[B(*, M)]_{2}=[M]_{2}+[\mathbb{R P}(\nu \oplus 1)]_{2},
$$

pois a soma conexa $X \# Y$ pode ser vista como um tipo especial de cirurgia da variedade $X \dot{\cup} Y$ e daí, pela invariança da classe de bordismo por cirurgia, obtemos $[X \# Y]_{2}=[X \dot{\cup} Y]_{2}=[X]_{2}+[Y]_{2}$.

Para o caso geral construiremos, como em [15], uma variedade $W$ cujo bordo é a união de três das variedades da definição do blow-up.

Lema 3.3.1. Seja $A$ uma subvariedade invariante fechada de uma $G$-variedade fechada $M^{n}$ e $\nu$ o fibrado normal de $A$ em $M$. Então existe uma variedade $W$ tal que

$$
\partial W=M \dot{\cup} B(A, M) \dot{\cup} \mathbb{R} P(\nu \oplus 1) .
$$

Prova. Construamos uma tal $(n+1)$-variedade como

$$
W=[M \times I \dot{\cup} \mathbb{R} P(\nu \oplus 1) \times I] \cup_{j} D(\nu) \times I,
$$

onde $I=[0,1]$ e $j$ é a inclusão de

$$
D(\nu) \times\{0\} \text { em } M \times\{0\}, \quad \text { e de } D(\nu) \times\{1\} \text { em } \mathbb{R P}(\nu \oplus 1) \times\{1\} .
$$


Temos então

$$
\begin{aligned}
\partial W= & {[\partial(M \times I) \dot{U} \partial(\mathbb{R} P(\nu \oplus 1) \times I)] \cup_{j} \partial(D(\nu) \times I) } \\
= & {[M \times\{0\} \dot{\cup} M \times\{1\} \dot{\cup} \mathbb{R} P(\nu \oplus 1) \times\{0\} \dot{U} \mathbb{R R P}(\nu \oplus 1) \times\{1\}] } \\
& \cup_{j} \partial(D(\nu) \times I) \\
= & M \times\{1\} \dot{\cup}\left\{[M \times\{0\} \dot{\cup} \mathbb{R} P(\nu \oplus 1) \times\{1\}] \cup_{j} \partial(D(\nu) \times I)\right\} \\
& \dot{U} \mathbb{I R P}(\nu \oplus 1) \times\{0\} \\
\equiv & M \dot{U} B(A, M) \dot{U} \mathbb{I R P}(\nu \oplus 1),
\end{aligned}
$$

onde $\partial(D(\nu) \times I)=S(\nu) \times I \cup_{S(\nu) \times\{0,1\}} D(\nu) \times\{0,1\}$ por [3, 13.13].

Geometricamente, considerando como no Exemplo 3.1.5 $M=S^{1}$ e $A=\{*\}$, temos

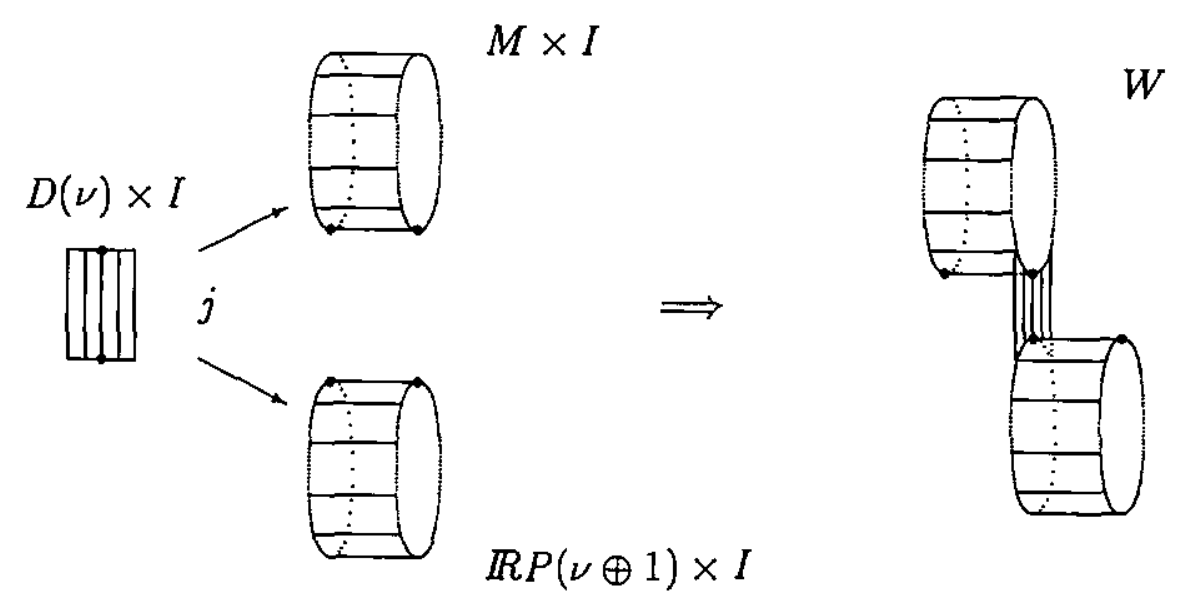

Em termos das classes de bordismo segue imediatamente do lema acima que

$$
[M]_{2}+[B(A, M)]_{2}+[\operatorname{IRP}(\nu \oplus 1)]_{2}=0
$$

Ou seja,

Proposição 3.3.2. Seja A uma subvariedade invariante fechada de uma G-variedade fechada $M^{n}$ e $\nu$ o fibrado normal de $A$ em $M$. Então

$$
[B(A, M)]_{2}=[M]_{2}+[I R P(\nu \oplus 1)]_{2}
$$


Casos especiais importantes da proposição acima aparecem quando consideramos o blow-up do conjunto dos pontos fixos $F$ de $M$.

Teorema 3.3.3. Seja $M^{n}$ uma G-variedade fechada com conjunto de pontos fixos $F=\operatorname{Fix}(G, M)=\dot{U} F_{i}=F_{1} \dot{U} \cdots \dot{U} F_{\mathrm{r}}$ e com fibrado normal denotado por $\nu=\nu(F, M)=\sum_{i=1}^{r} \nu\left(F_{i}, M\right)=\sum_{i=1}^{r} \nu_{i}$. Então

$$
[B(F, M)]_{2}=[M]_{2}+[\mathbb{R P}(\nu \oplus 1)]_{2} .
$$

Prova. Temos

$$
\begin{aligned}
B(F, M) & =B\left(F_{r}, B\left(F_{r-1}, \ldots B\left(F_{2}, B\left(F_{1}, M\right)\right) \ldots\right)\right) \\
& =\left(\ldots\left(\left(M \underset{F_{1}}{\#} \mathbb{R} P\left(\nu_{1} \oplus 1\right)\right) \underset{F_{2}}{\#} \mathbb{R} P\left(\nu_{2} \oplus 1\right)\right) \underset{F_{3}}{\# \ldots)} \underset{F_{r}}{\#} \mathbb{R} P\left(\nu_{r} \oplus 1\right) .\right.
\end{aligned}
$$

Por indução em $r$, usando o Lema 3.3.1, obtemos

$$
[B(F, M)]_{2}=[M]_{2}+\sum_{i=1}^{r}\left[\mathbb{R} P\left(\nu_{i} \oplus 1\right)\right]_{2}=[M]_{2}+[\mathbb{R P}(\nu \oplus 1)]_{2} .
$$

Podemos então relacionar a classe de bordismo do blow-up do conjunto dos pontos fixos $F$ em $M$ com as classes de bordismo dos blow-ups dos $F_{i}$ 's em $M$.

Corolário 3.3.4. Seja $M^{n}$ uma $G$-variedade fechada com conjunto de pontos fixos $F=\operatorname{Fix}(G, M)=\dot{U} F_{i}=F_{1} \dot{\cup} \cdots \dot{U} F_{r}$, com cada $F_{i}$ conexo, e fibrado normal $\nu=\nu(F, M)=\sum_{i=1}^{r} \nu\left(F_{i}, M\right)=\sum_{i=1}^{r} \nu_{i}$. Então:

a) se $r$ é impar temos

$$
[B(F, M)]_{2}=\sum_{i=1}^{r}\left[B\left(F_{i}, M\right)\right]_{2}
$$

b) ser é par temos

$$
[B(F, M)]_{2}=[M]_{2}+\sum_{i=1}^{r}\left[B\left(F_{i}, M\right)\right]_{2} .
$$


Prova. Pelo Teorema 3.3.3 temos

$$
[B(F, M)]_{2}=[M]_{2}+\sum_{i=1}^{r}\left[\operatorname{IRP}\left(\nu_{i} \oplus 1\right)\right]_{2} .
$$

Da Proposição 3.3.2 obtemos

$$
\left[\mathbb{R P}\left(\nu_{i} \oplus 1\right)\right]_{2}=[M]_{2}+\left[B\left(F_{i}, M\right)\right]_{2},
$$

que substituído na equação anterior fornece o resultado.

Quando consideramos $G=\mathbb{Z}_{2}$ a classe de bordismo do blow-up dos pontos fixos é nula, ou seja,

Teorema 3.3.5. Se $M^{n}$ é uma $\mathbb{Z}_{2}$-variedade fechada com conjunto dos pontos fixos $F=\operatorname{Fix}\left(\mathbb{Z}_{2}, M\right)$, então $[B(F, M)]_{2}=0$.

Prova. Pelo Teorema de Conner-Floyd, 2.5.2, temos $[M]_{2}=[\operatorname{RP}(\nu \oplus 1)]_{2}$. Daí, usando o Teorema 3.3.3 obtemos o resultado.

Como aplicações do teorema 3.3.5 temos

Corolário 3.3.6. Se $M^{n}$ é uma variedade fechada de dimensão par, com $[M]_{2}=0$, então não existe involução sobre $M$ com conjunto de pontos fixos $F$ igual a um número impar de pontos isolados.

Prova. Suponha que exista uma tal involução com $2 k+1$ pontos fixos isolados, $x_{i}$, em $M^{n}$. Como $\mathbb{R P}\left(\nu_{i} \oplus 1\right)=\mathbb{R} P^{n}$, para cada $\nu_{i}=\nu_{i}\left(x_{i}, M^{n}\right)$, temos pelo Teorema 3.3.3

$$
[B(F, M)]_{2}=[M]_{2}+\sum_{i=1}^{2 k+1}\left[R P^{n}\right]_{2} .
$$

Daí, como $[M]_{2}=0$ e $n$ é par, obtemos $[B(F, M)]_{2} \neq 0$, que é um absurdo pelo Teorema 3.3.5. 
Corolário 3.3.7. Se $M$ é uma variedade fechada, com $[M]_{2} \neq 0$, então não existe involução sobre $M$ com conjunto de pontos fixos $F$ igual a um número par de pontos isolados.

Prova. Como na prova anterior, supondo que $\mathrm{F}$ tenha $2 k$ pontos, temos

$$
\left[B\left(F, M^{n}\right)\right]_{2}=[M]_{2}+\sum_{i=1}^{2 k}\left[\operatorname{IRP} P^{n}\right]_{2}=[M]_{2} \neq 0,
$$

que é um absurdo pelo Teorema 3.3.5.

Em particular, para $r \geq 1$, temos:

1) Não existe involução sobre $S^{2}, \quad\left(T^{2}\right)^{r}$ ou $(K b)^{r}$ com conjunto de pontos fixos igual a um ponto.

2) Não existe involução sobre $\left(I R P^{2}\right)^{2 r-1}$ com conjunto de pontos fixos igual a dois pontos.

Outro caso especial importante a considerar é quando o conjunto de pontos fixos da $G$-variedade $M$ tem dimensão 1 . Vejamos inicialmente a classe de bordismo de $\mathbb{R} P(\xi \oplus 1)$.

Dado um fibrado vetorial $\mathbb{R}^{k} \rightarrow \xi \rightarrow B^{m}$, podemos ter $[\operatorname{IRP}(\xi \oplus 1)]_{2}=0 \mathrm{com}$ $[B]_{2} \neq 0$. Considerando, por exemplo, um fibrado de dimensão ímpar sobre um ponto, $\quad \mathbb{R}^{2 k+1} \rightarrow \xi \rightarrow\{*\}$, temos $\mathbb{R} P(\xi \oplus 1)=\mathbb{R} P^{2 k+1}$, isto é, $[B]_{2} \neq 0$ $\operatorname{com}[\operatorname{IRP}(\xi \oplus 1)]_{2}=0$.

No entanto, se $\mathbb{R}^{k} \rightarrow \xi \rightarrow B^{m}$ é um fibrado trivial em que o espaço base borda, então $\operatorname{IRP}(\xi \oplus 1)$ borda. De fato, como $[B]_{2}=0$ existe $W^{m+1}$ tal que $\partial W=B$. Mais ainda, como $\xi \cong B \times \mathbb{R}^{k}$ temos $\mathbb{R} P(\xi \oplus 1) \cong B \times \mathbb{R} P^{k}$. Assim

$$
\partial\left(W \times \mathbb{R} P^{k}\right)=\partial W \times \mathbb{R} P^{k}=B \times \mathbb{R} P^{k} \cong \mathbb{R} P(\xi \oplus 1),
$$

mostrando que $\mathbb{R} P(\xi \oplus 1)$ borda.

Para o caso particular de fibrado não trivial com espaço base igual a $\mathbb{R} P^{1}$ utilizamos o lema seguinte cuja referência é Conner-Floyd [5, Lema 2.2]. 
Lema 3.3.8. Se $\mathbb{R} \rightarrow \gamma_{1}^{1} \rightarrow \mathbb{R} P^{1}$ é o fibrado linha canônico não-trivial $e$ $\mathbb{R}^{n} \rightarrow \varepsilon^{n} \rightarrow \mathbb{R} P^{1}$ é o fibrado trivial $n$-dimensional, então para cada $n \geq 0$ temos $\left[\operatorname{IRP}\left(\gamma_{1}^{1} \oplus \varepsilon^{n}\right)\right]_{2}=0 \in \mathcal{N}_{n+1}$.

Temos então

Proposição 3.3.9. Se $\mathbb{R}^{k} \rightarrow \xi \rightarrow \mathbb{R} P^{1}$ é um fibrado vetorial $k$-dimensional não orientável, então $[\operatorname{IRP}(\xi \oplus 1)]_{2}=0$.

Prova. Se $\xi \oplus 1$ é trivial vimos acima que $\mathbb{R} P(\xi \oplus 1)$ borda. Se $\xi \oplus 1$ é não-trivial então $\xi \oplus 1 \cong \gamma_{1}^{1} \oplus \varepsilon^{k-1}$ e o resultado segue do Lema 3.3.8.

Segue então que

Teorema 3.3.10. Seja $M^{n}$ uma G-variedade fechada com conjunto de pontos fixos $F=\dot{\cup} F_{i}=F_{1} \dot{\cup} \cdots \dot{U} F_{r}$ de dimensão 1. Então

$$
\left[B\left(F_{i}, M\right)\right]_{2}=[M]_{2}=[B(F, M)]_{2}
$$

Prova. Como $\operatorname{dim} F_{i}=1$ temos, para todo i, $F_{i}=\mathbb{R} P^{1}=S^{1}$. Considerando o fibrado $(n-1)$-dimensional $\mathbb{R}^{n-1} \rightarrow \nu_{i} \rightarrow F_{i}$ e o fibrado projetivo associado $\mathbb{R} P^{n-1} \rightarrow \mathbb{R} P\left(\nu_{i} \oplus 1\right) \rightarrow F$; temos pela Proposição 3.3 .9 que $\left[\mathbb{R} P\left(\nu_{i} \oplus 1\right)\right]_{2}=0$.

Mas pela Proposição 3.3.2 e pelo Teorema 3.3.3 temos

$$
[M]_{2}+\left[B\left(F_{i}, M\right)\right]_{2}+\left[\operatorname{IRP}\left(\nu_{i} \oplus 1\right)\right]_{2}=0
$$

e

$$
[M]_{2}+[B(F, M)]_{2}+[\mathbb{R P P}(\nu \oplus 1)]_{2}=0
$$

Daí,

$$
\left[B\left(F_{i}, M\right)\right]_{2}=[M]_{2}=[B(F, M)]_{2}
$$

Corolário 3.3.11. Seja $M^{n}$ é uma variedade fechada com $[M]_{2} \neq 0$. Entāo não existe involução sobre $M$ com conjunto de pontos fixos $F$ tendo dimensão 1. 
Prova. Se existisse uma tal involução, pelo Teorema $3.3 .10 \operatorname{com} G=\mathbb{Z}_{2}$ e pelo Teorema 3.3.5, teríamos $[M]_{2}=[B(F, M)]_{2}=0$, que é um absurdo.

Em particular, não existe involução sobre $\left(\mathbb{R} P^{2}\right)^{2 r-1}$ com conjunto de pontos fixos igual a $S^{1}$.

Outro caso especial importante de blow-up a analisar é quando o conjunto de pontos fixos de uma $\mathbb{Z}_{2}$-variedade tem codimensão 1 . Este caso é considerado na seção seguinte. 


\subsection{Bordismo de Variedades Não-Singulares}

Para tratar de variedades não-singulares usaremos o lema seguinte cuja referência é Conner-Floyd [5, Lema 2.1].

Lema 3.4.1. Se $\xi \rightarrow V^{m}$ é um fibrado vetorial diferenciável 2-dimensional sobre uma variedade fechada, então $[\operatorname{IRP}(\xi)]_{2}=0 \in \mathcal{N}_{m+1}$.

Prova. Pelo Teorema de Borel-Hirzebruch, 2.4.1, como $k=2$, temos

$$
c^{2}=c p^{*}\left(v_{1}\right)+p^{*}\left(v_{2}\right), \quad \text { onde } \quad v_{i}=w_{i}\left(\xi \rightarrow V^{n}\right)
$$

que aplicado a

$$
w\left(\xi_{2}\right)=(1+c)^{2}+(1+c) p^{*}\left(v_{1}\right)+p^{*}\left(v_{2}\right) \bmod 2
$$

fornece

$$
w\left(\xi_{2}\right)=1+p^{*}\left(v_{1}\right) \quad \bmod 2 .
$$

Daí, denotando $w_{i}=w_{i}\left(V^{m}\right)$, temos

$$
\begin{aligned}
w(\operatorname{IRP}(\xi)) & =\left[1+p^{*}\left(w_{1}\right)+\cdots+p^{*}\left(w_{m}\right)\right] \cdot\left[1+p^{*}\left(v_{1}\right)\right] \\
& =\left[1+p^{*}\left(w_{1}\right)+\cdots+p^{*}\left(w_{m}\right)\right]+\left[p^{*}\left(v_{1}\right)+p^{*}\left(w_{1} v_{1}\right)+\cdots+p^{*}\left(w_{m} v_{1}\right)\right] \\
& =1+p^{*}\left(w_{1}+v_{1}\right)+p^{*}\left(w_{2}+w_{1} v_{1}\right)+\ldots+p^{*}\left(w_{m}+w_{m-1} v_{1}\right)+p^{*}\left(w_{m} v_{1}\right)
\end{aligned}
$$

ou seja,

$$
w_{i}(\operatorname{IRP}(\xi))=p^{*}\left(w_{i}+w_{i \rightarrow 1} v_{1}\right)
$$

Considerando $I=\left(i_{1}, \ldots, i_{r}\right)$ uma partição de $m+1=\operatorname{dim} I R P(\xi)$ temos então que o monômio $w_{i_{1}}(I R P(\xi)) \ldots w_{i_{r}}(I R P(\xi))$ é a imagem por $p^{*}$ de uma soma de termos $(m+1)$-dimensionais, envolvendo apenas os $w_{i}$ 's e $v_{1}$, provenientes de $H^{m+1}\left(V^{m}, \mathbb{Z}_{2}\right)=0$. Assim todo número de Stiefel-Whitney $W_{l}(\operatorname{IRP}(\xi))$ é nulo e portanto $\operatorname{IRP}(\xi)$ borda. 
Em particular, se $\xi$ é um fibrado linha temos $[R P(\xi \oplus 1)]_{2}=0$.

Temos então que $\mathbb{Z}_{2}$-variedades não-singulares bordam, isto é,

Teorema 3.4.2. Seja $M$ uma $\mathbb{Z}_{2}$-variedade não-singular com conjunto de pontos fixos $F=\dot{\cup} F_{i}=F_{1} \dot{\cup} \cdots \dot{U} F_{r}$. Então, para todo $i$,

$$
\left[B\left(F_{i}, M\right)\right]_{2}=[M]_{2}=\left[\operatorname{RRP}\left(\nu_{i} \oplus 1\right)\right]_{2}=0 .
$$

Prova. Como $M$ é não-singular temos $\operatorname{codim} F_{i}=1$, e assim os fibrados normais $\nu_{i}=\nu_{i}\left(F_{i}, M\right)$ são fibrados linha. Pelo Lema 3.4.1 temos que $\left[\operatorname{IRP}\left(\nu_{i} \oplus 1\right)\right]_{2}=0$ e pelo Teorema 3.3.5 temos $[B(F, M)]_{2}=0$. Daí, pelo Teorema 3.3.3 e pela Proposição 3.3.2, obtemos $[M]_{2}=[B(F, M)]_{2}=0$ e $\left[B\left(F_{i}, M\right)\right]_{2}=0$.

Corolário 3.4.3. Seja $M^{n}$ é uma variedade fechada com $[M]_{2} \neq 0$. Então não existe involuçãa sobre $M$ com conjunto de pontos fixos $F$ tendo codimensão 1 .

Prova. Se existisse uma tal involução, denotando $F=\dot{U} F_{i}$, teríamos pelo Teorema 3.4.2 $\left[B\left(F_{i}, M\right)\right]_{2}=[M]_{2}=0$, que é um absurdo.

Em particular, obtemos novamente que não existe involução sobre $\left(\mathbb{R} P^{2}\right)^{2 r-1}$ com conjunto de pontos fixos igual a $S^{1}$. Observamos contudo que $S^{1}$ pode estar no conjunto de pontos fixos de $\mathbb{I R} P^{2}$.

Exemplo 3.4.4. Considere o espaço projetivo real 2-dimensional

$$
\mathbb{R} P^{2}=\left\{\left[x_{0}, x_{1}, x_{2}\right] ;\left(x_{0}, x_{1}, x_{2}\right) \in \mathbb{R}^{3}-\{0\}\right\},
$$

onde $\left[x_{0}, x_{1}, x_{2}\right]=\left\{\lambda\left(x_{0}, x_{1}, x_{2}\right) ; \lambda \in \mathbb{R}-\{0\}\right\}$ são as coordenadas homogêneas de um ponto. Defina uma involução $T$ sobre $\mathbb{R} P^{2}$ por

$$
\left[x_{0}, x_{1}, x_{2}\right] \rightarrow T\left(\left[x_{0}, x_{1}, x_{2}\right]\right)=\left[-x_{0},-x_{1}, x_{2}\right] .
$$

Temos então

$$
F=\operatorname{Fix}\left(T, \mathbb{R} P^{2}\right)=\left\{\left[0,0, x_{2}\right]\right\} \dot{\cup}\left\{\left[x_{0}, x_{1}, 0\right]\right\} \simeq P \dot{\cup} S^{1},
$$


onde $P$ denota o ponto $\left[0,0, x_{2}\right]$. (Observe que $\left[x_{0}, x_{1}, 0\right]=-\left[x_{0}, x_{1}, 0\right]$.)

Observemos que $\mathbb{R} P^{2}$ pode se transformar numa variedade não-singular através de um blow-up no ponto $P$, isto é,

$$
B\left(P, \mathbb{R} P^{2}\right)=\mathbb{R} P^{2} \underset{\{P\}}{\#} \mathbb{R} P(\nu \oplus 1)=\mathbb{R} P^{2} \# \mathbb{R} P^{2}=K b
$$

Em [4, Capítulo V], Conner e Floyd obtêm poucos resultados sobre ações de $\left(\mathbb{Z}_{2}\right)^{k}=\mathbb{Z}_{2} \times \cdots \times \mathbb{Z}_{2}, \quad k$-termos, em uma variedade diferenciável $M^{n}$. Resumidamente eles provam:

1) $\operatorname{Se}\left(\mathbb{Z}_{2}\right)^{k}$ atua diferenciavelmente numa variedade fechada $M^{n}$ sem pontos fixos, então $\left[M^{n}\right]_{2}=0$.

2) Se $\mathbb{Z}_{2} \times \mathbb{Z}_{2}$ atua diferenciavelmente numa variedade fechada $M^{n}$ com pontos fixos isolados, então $\left[M^{n}\right]_{2}=0$ ou $n=2 m$ com $\left[M^{n}\right]_{2}=\left[\left(\mathbb{R} P^{2}\right)^{m}\right]_{2}$ (aqui, denotamos $\left(\mathbb{R} P^{2}\right)^{m}=\mathbb{R} P^{2} \times \cdots \times \mathbb{R} P^{2}, m$ termos $)$.

3) Não existe ação diferenciável de $\left(\mathbb{Z}_{2}\right)^{k}$ sobre uma variedade fechada $M^{n}, n>0$, tendo conjunto de pontos fixos igual a um único ponto.

Estes resultados não se generalizam, conforme vemos no exemplo abaixo.

Exemplo 3.4.5. Consideremos uma aplicação diferenciável $T: D^{2 k} \rightarrow D^{2 k}$ definida por $T\left(z_{1}, \ldots, z_{k}\right)=\left(i z_{1}, \ldots, i z_{k}\right)$, onde $i=\sqrt{-1}$. Identificando os pontos antipodais do bordo do disco $D^{2 k}$, obtemos $T: \mathbb{R} P^{2 k} \rightarrow \mathbb{R} P^{2 k}$ de periodo 4 (e portanto uma ação de $\mathbb{Z}_{4}$ sobre $\mathbb{R} P^{2 k}$ ) com exatamente 1 ponto fixo.

Em particular obtemos ações de $\mathbb{Z}_{\mathbf{4}}$ sobre $\mathbb{R} P^{4}$ e sobre $\mathbb{R} P^{2} \times \mathbb{R} P^{2}$ tendo cada uma exatamente 1 ponto fixo. Dai, fazendo um blow-up sobre cada ponto fixo, obtemos variedades

$$
B\left(*, \mathbb{R} P^{4}\right)=\mathbb{R} P^{4} \# \mathbb{R} P^{4} \quad \text { e } \quad B\left(*, \mathbb{R} P^{2} \times \mathbb{R} P^{2}\right)=\left(\mathbb{R} P^{2} \times \mathbb{R} P^{2}\right) \# \mathbb{R} P^{4},
$$

sobre as quais $\mathbb{Z}_{4}$ atua sem pontos fixos. Mais ainda,

$$
\left[B\left(*, \mathbb{R} P^{4}\right)\right]_{2}=\left[\mathbb{R} P^{4}\right]_{2}+\left[\mathbb{R} P^{4}\right]_{2}=0
$$




$$
\left[B\left(*, \mathbb{R} P^{2} \times \mathbb{R} P^{2}\right)\right]_{2}=\left[\left(\mathbb{R} P^{2} \times \mathbb{R} P^{2}\right)\right]_{2}+\left[\mathbb{R} P^{4}\right]_{2} \neq 0
$$

Em termos de $\left(\mathbb{Z}_{2}\right)^{k}$-ação temos o seguinte resultado:

Teorema 3.4.6. Se $\left(\mathbb{Z}_{2}\right)^{k} \times M^{k+1} \rightarrow M^{k+1}$ é uma ação não-singular semi-livre, com conjunto de pontos fixos $F$ e $\nu=\nu(F, M)$, então

$$
[F]_{2}=0, \quad[B(F, M)]_{2}=[M]_{2} \quad \text { e }[\mathbb{I R P}(\nu \oplus 1)]_{2}=0
$$

Prova. Como a ação é semi-livre, denotando $G=\left(\mathbb{Z}_{2}\right)^{k}$, temos $G_{x}=G$ ou $G_{x}=\{e\}$. Assim $M(G)=\operatorname{Fix}(G, M)=F$ e $M(e)=M-M(G)$. Como a ação é nāo-singular devemos ter

$$
\operatorname{dim}_{\mathbf{Z}_{2}} G=k=\operatorname{codim} M(G)=\operatorname{codim} F
$$

e

$$
\operatorname{dim}_{\mathbb{Z}_{2}}\{e\}=0=\operatorname{codim} M(e),
$$

ou seja, $\operatorname{dim} F=1$. Como o conjunto de pontos fixos é uma variedade fechada, devemos ter $F=\dot{U} S^{1}$, de modo que $[F]_{2}=0$. Daí, pelo Teorema 3.3.10, obtemos $[B(F, M)]_{2}=[M]_{2}$. Pelo Teorema 3.3 .3 obtemos $[I R P(\nu \oplus 1)]_{2}=0$. 


\section{Referências Bibliográficas}

[1] BOREL, A. and HIRZEBRUCH, F. On characteristic classes of homogeneous spaces; I. American Journal of Mathematics, 80 (1958), 458-538; II. American Journal of Mathematics, 81 (1959), 315-382.

[2] BREDON, G. E. Introduction to Compact Transformation Groups. Princeton University Press, 1972.

[3] BROCKER, T. and JANICH, K. Introduction to Differential Topology. Cambridge University Press, 1982.

[4] CONNER, P. E. and FLOYD, E. E. Differentiable Periodic Maps. Ergebnisse der Mathematic, vol. 33, Springer-Verlag, 1964.

[5] CONNER, P. E. and FLOYD, E. E. Fibring whithin a cobordism class. Michigan Mathematical Journal, 12 (1965), 33-47.

[6] KAWAKUBO, K. The Theory of Transformation Groups. Oxford University Press, 1991.

[7] KOSCHORKE, U. Vector Fields an Other Vector Bundle Morphisms - A Singularity approach. Lecture Notes in Mathematics, 847, Springer-Verlag, 1981.

[8] MASSEY, W. S. Algebraic Topology: An Introduction. Harcourt, Brace \& World, Inc., New York, 1967.

[9] MASSEY, W. S. Singular Homology Theory. Graduate Texts in Mathematics, 70, Springer-Verlag, 1980. 
[10] MILNOR, J. A procedure for killing homotopy groups of differentiable manifolds. Proceedings of Symposia in Pure Mathematics (Differential Geometry), American Mathematical Society, volume 3, (1961), 39-55.

[11] MILNOR, J; W. and.STASHEFF, J. D. Characteristic Classes. Princeton University Press, 1974.

[12] STEENROD, N. E. The Topology of Fibre Bundles. Princeton University Press, 1951.

[13] THOM, R. Quelques propriétés globales des variétés différentiables. Commentarii Mathematici Helvetici, 28 (1954), 17-86.

[14] WASSERMAN, A. G. Symplifying group actions. Topology and its Applications, 75 (1997), 13-31.

[15] WASSERMAN, A. G. Relations among characteristic classes and fixed points $I$ - The recognition principle. (preprint). 


\section{ERRATA}

Página Local Erro

(Resumo) linha 1 de Lie $G$

(Resumo) linha 1 variedade $M$,

(Abstract) linha 1 a Lie Group $G$

(Abstract) linha 1 a manifold $M$,

i linha $6 G$-variedade $M$,

i linha $10 G$-variedade $M$,

i linha 12 utiliza simplificar

ii linha $5 \quad M$ obtemos

ii linha 16 Conner Floyd

ii linha 16 condição para

3 linha $2 x \in G$

5. linha $13(H) \leq(H)$,

$5 \quad$ linha $15\left(\frac{\vec{G}}{H}\right) \rightarrow H$

$8 \quad \operatorname{linha} 3 \frac{G}{G}$

$8 \quad$ linha $4 \frac{G}{\{e\}}$

$8 \quad$ linha $6 \quad \mathrm{G} / \mathrm{H}$

8 linha $8 \quad \frac{G}{H}$

$9 \quad$ linha $7 \quad \Phi_{g}$

9 linha 25 para $\mathrm{x}$

11 linha 5 no acima

15 linha 7 correspondente a $F_{b}(\eta)$

18 linha 5 Conner-Floyd 2.5.2 que

$18 \quad$ linha 9 classse

19 linha 14 conjunto os

21 linha $18 \quad[G, M]_{2}$

28 linha $11 \chi(M)$.

31 linha 10 e $\tau_{V}$, temos

$31 \quad$ (diagr.) $\tau_{V}$

\section{Correção}

de Lie compacto $G$

variedade diferenciável compacta $M$,

a compact Lie group $G$

a compact differentiable manifold $M$,

$G$-variedade diferenciável $M$,

$G$-variedade $M$ e $\nu=\nu(A, M)$,

utiliza para simplificar

$M$, obtemos

Conner-Floyd

condição necessária para

$x \in X$

$(H) \leq(K)$,

$\left(\frac{G}{H}\right) \rightarrow(H)$

$\left(\frac{G}{G}\right)$

$\left(\frac{G}{e}\right)$

$\left(\frac{G}{H}\right)$

$\left(\frac{G}{H}\right)$

$\Phi(g)$

para todo $\mathrm{x}$

no diagrama acima

correspondente $F_{b}(\eta)$

Conner-Floyd, 2.5.2, que

classe

conjunto dos

$\left[G, M^{n}\right]_{2}$

$\chi(M)$ :

e $\tau_{V^{n}}$, temos

$\tau_{V n}$ 
Página Local Erro

$32 \quad$ linha $16 s=1,2, \ldots, k$,

32 linha $22 p^{*}\left(v_{p}\right)$

35 linha $18[T, S(\nu)]_{2}$ borda,

$38 \quad$ linha $8 \quad F=\operatorname{Fix}\left(\mathbb{Z}_{2}, M\right)$

38 linha 10 Lema 2.6.3, o

38 linha 111 , e assim

40 linha 10 variedade $V$

40 linha 11 componentes.

41 linha $6 \quad \mathbb{Z}_{2}$-variedade tendo

41 linha 7 codimensional $V$ com

42 linha 6 não-singulares.

\section{Correção}

$s=0,1,2, \ldots, k$,

$p^{*}\left(v_{s}\right)$

$(T, S(\nu))$ borda,

$\operatorname{com} F=\operatorname{Fix}\left(\mathbb{Z}_{2}, M\right)$

Lema $2.6 .3 \mathrm{o}$

$n-1$, e assim

variedade diferenciável compacta $V$

componentes conexas.

$\mathbb{Z}_{2}$-variedade $V$ tendo

codimensional com

não-singulares.(Conforme estabelecido

no início do Capítulo II, aqui também

variedade significa variedade

diferenciável compacta.)

44 linha 2 temos

temos as variedades (diferenciáveis)

em $\mathrm{Kb}$ respectivamente.

48 linha 3 em Kb.

$\dot{\cup}_{i=1}^{r}$

$\left(G_{(x, y, z)}\right)=G_{(x, y, z)}$

Exemplo 3.1.2

$\dot{\cup}_{i=1}^{r}$

$\dot{U}_{i=1}^{r}$

$\nu_{i}=\nu\left(x_{i}, M^{n}\right)$

$\varepsilon^{k}$

$M^{n}$ uma

$\left(\mathbb{R} P^{2}\right)^{2 r-1}=\mathbb{R} P^{2} \# \cdots \# \mathbb{R} P^{2}$

$V^{m}$

não-singular fechada

$M^{n}$ uma

within

Approach 


\section{ADENDO}

Conforme sugestão da Comissão Julgadora listamos abaixo, como um adendo à Introdução, os principais resultados obtidos neste trabalho:

Teorema 3.3.3 Seja $M^{n}$ uma G-variedade fechada com conjunto de pontos fixos $F=\operatorname{Fix}(G, M)=\dot{U} F_{i}=F_{3} \dot{U} \ldots \dot{U} F_{r}$ e com fibrado normal denotado por $\nu=\nu(F, M)=\dot{U}_{i=1}^{r} \nu\left(F_{i}, M\right)=\dot{U}_{i=1}^{r} \nu_{i}$. Então

$$
[B(F, M)]_{2}=[M]_{2}+[\operatorname{IRP}(\nu \oplus 1)]_{2}
$$

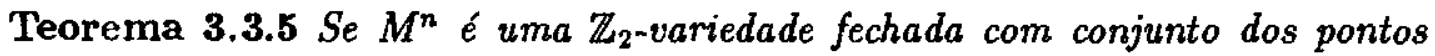
fixos $F=\operatorname{Fix}\left(\mathbb{Z}_{2}, M\right)$, então $[B(F, M)]_{2}=0$.

Teorema 3.3.10 Seja $M^{\mathrm{n}}$ uma G-variedade fechada com conjunto de pontos fixos $F=\dot{U} F_{i}=F_{1} \dot{U} \ldots \dot{U} F_{r}$ de dimensão 1. Então.

$$
\left[B\left(F_{i}, M\right)\right]_{2}=[M]_{2}=[B(F, M)]_{2}
$$

Teorema 3.4.2 Seja $M$ uma $\mathbb{Z}_{2}$-variedade nẫo-singular fechada com conjunto de pontos fixos $F=\dot{\cup} F_{i}=F_{1} \dot{U} \ldots \dot{\cup} F_{r}$. Então, para todo $i$,

$$
\left[B\left(F_{i}, M\right)\right]_{2}=[M]_{2}=\left[\operatorname{IRP}\left(\nu_{i} \oplus 1\right)\right]_{2}=0
$$

Teorema 3.4.6 Se $\left(\mathbb{Z}_{2}\right)^{k} \times M^{k+1} \rightarrow M^{k+1}$ é uma ação não-singular semi-livre, com conjunto de pontos fixos $F$ e $\nu=\nu(F, M)$, então

$$
[F]_{2}=0, \quad[B(F, M)]_{2}=[M]_{2} \quad \text { e } \quad[\mathbb{R R P}(\nu \oplus 1)]_{2}=0
$$

Esclarecemos ainda que, neste trabalho, os resultados obtidos estão em termos de bordismo módulo 2. Ainda não obtivemos resultados significativos na classe equivariante. 